\title{
Amphibians and reptiles of the state of Chihuahua, Mexico, with comparisons with adjoining states
}

\author{
Julio A. Lemos-Espinal', Geoffrey R. Smith², \\ Guillermo A. Woolrich-Piña ${ }^{3}$, Alexander Cruz ${ }^{4}$
}

I Laboratorio de Ecologia-UBIPRO, FES Iztacala UNAM. Avenida los Barrios 1, Los Reyes Iztacala, Tlalnepantla, edo. de México, Mexico - 540902 Department of Biology, Denison University, Granville, OH, USA 430233 Instituto Tecnológico Superior de Zacapoaxtla. Carretera Acuaco-Zacapoaxtla Km. 8, Col. Totoltepec C. P. 73680, Zacapoaxtla, Puebla, Mexico 4 Department of Ecology and Evolutionary Biology (EBIO), University of Colorado - Boulder, Campus Box 334 UCB, Boulder, CO USA 80309-0334

Corresponding author: Guillermo A. Woolrich-Piña (gwoolrich@live.itsz.edu.mx)

Academic editor: J. Penner | Received 27 September 2016 | Accepted 31 January 2017 | Published 28 February 2017

http://zoobank.org/4FC0422E-BE90-4A1D-8AEF-2608FBA8A28D

Citation: Lemos-Espinal JA, Smith GR, Woolrich-Piña GA, Cruz A (2017) Amphibians and reptiles of the state of Chihuahua, Mexico, with comparisons with adjoining states. ZooKeys 658: 105-130. https://doi.org/10.3897/ zookeys.658.10665

\begin{abstract}
Chihuahua is Mexico's largest state, and its physiographic complexity affects the distribution of its herpetofauna. We list amphibians and reptiles for the state of Chihuahua, with their conservation status. We also compare this list to those of six adjoining states in the United States and Mexico (New Mexico, Texas, Coahuila, Durango, Sinaloa, and Sonora). A total of 175 species of amphibians and reptiles is found in Chihuahua. Thirty-eight are amphibians, and 137 reptiles. Chihuahuan amphibians and reptiles represent just over $37 \%$ of such species from Chihuahua and neighboring states. Chihuahua shares the highest proportion of its herpetofauna with Sonora and Durango. Most of the herpetofauna of Chihuahua falls in IUCNs least concern category and is not listed by SEMARNAT. However, turtles in Chihuahua are a group of particular conservation concern.
\end{abstract}

\section{Keywords}

Checklist, Chihuahuan Desert, conservation status, herpetofauna, Sierra Madre Occidental 


\section{Introduction}

Chihuahua is the largest state in Mexico. Its $245,612 \mathrm{~km}^{2}$ (lying between $25^{\circ} 38^{\prime} \mathrm{N}$ to the south, $31^{\circ} 47^{\prime} \mathrm{N}$ to the north, and between $103^{\circ} 18^{\prime} \mathrm{W}$ to the east, and $109^{\circ} 7^{\prime} \mathrm{W}$ to the west) represent $12.6 \%$ of the total territory of the nation. Chihuahua is physiographically complex (Fig. 1), and this complexity affects the distribution of the herpetofauna.

The western part of the state is primarily occupied by the Sierra Madre Occidental, which passes the Continental Divide, separating the Pacific and Atlantic drainages. In Chihuahua, the Sierra Madre varies in width from -130-160 km in the south (west of Hidalgo de Parral) to $-65-80 \mathrm{~km}$ in the north (west of Casas Grandes) (Tanner 1985, Lemos-Espinal and H. Smith 2007). The topography of the Sierra Madre Occidental of Chihuahua is very heterogeneous. The highest altitude is on Cerro Mohinora, at 3,300 $\mathrm{m}$, and the extreme southwestern Pacific slopes of this Sierra are characterized by deep canyons that drop down to $-250 \mathrm{~m}$ in the Barranca del Septentrión/Cañón de Chínipas, making diverse habitats for plants and animals. Copper Canyon is a $64,750 \mathrm{~km}^{2}$ system of six interconnected canyons located in Southwestern Chihuahua. Four of these six canyons are deeper than the Grand Canyon, some by over $305 \mathrm{~m}$. The deepest canyon is Urique Canyon, 1,870 m in depth; Batopilas Canyon is 1,830 m deep; Sinforosa Canyon is 1,800 m deep; and Copper Canyon is $1,759 \mathrm{~m}$ deep (Martin et al.1998, INEGI 2004, Wyndham 2004, Lemos-Espinal and H. Smith 2007, 2015a, LemosEspinal et al. 2013, http://www.earlham.edu/-garcier/Geology/coppercanyon.htm).

Over half of the state of Chihuahua, east of the Sierra Madre, is covered by high plains at $-1,200-1,700 \mathrm{~m}$. From these plains arise a large number of small to medium-sized, isolated sierras, some of which reach altitudes of over 2,000 m. Some are high enough to support coniferous forests, constituting continental "islands" surrounded by a "sea" of semiarid plains, where differentiation among populations is enhanced by isolation.

In extreme northeastern Chihuahua, deep canyons, similar to those on the Pacific side of the Sierra Madre Occidental, cut into the edge of the high plains, and support their own distinct herpetofaunal assemblages. Among them is the great Cañón de Santa Elena, in the Zona de Protección de Flora y Fauna Silvestre Cañón de Santa Elena, an extension of the Big Bend National Park of the United States.

In recent years, there has been a considerable increase in the study of Mexican regional and state herpetofaunas such as Sinaloa (Hardy and McDiarmid 1969), Peninsula of Baja California (Grismer 2002), Peninsula of Yucatan (Lee 1996), the Valley of Mexico (Ramírez-Bautista et al. 2009), Aguascalientes (McCranie and Wilson 2001), Chihuahua and Coahuila (Lemos-Espinal and H. Smith 2007a, b, 2015a, b; Lemos-Espinal and G. Smith 2016), Querétaro (Dixon and Lemos-Espinal 2010), San Luis Potosí (Lemos-Espinal and Dixon 2013), Michoacán (Alvarado-Díaz et al. 2015), Chiapas (Johnson et al. 2015b), Oaxaca (Mata-Silva et al. 2015), Nayarit (WoolrichPiña et al. 2016), Nuevo León (Lemos-Espinal et al. 2016), Sonora (Enderson et al. 2009, Lemos-Espinal and Rorabaugh 2015, Lemos-Espinal et al. 2015, Rorabaugh 2008, Rorabaugh and Lemos-Espinal 2016), and Tamaulipas (Farr 2015, Terán-Juárez et al. 2016). 


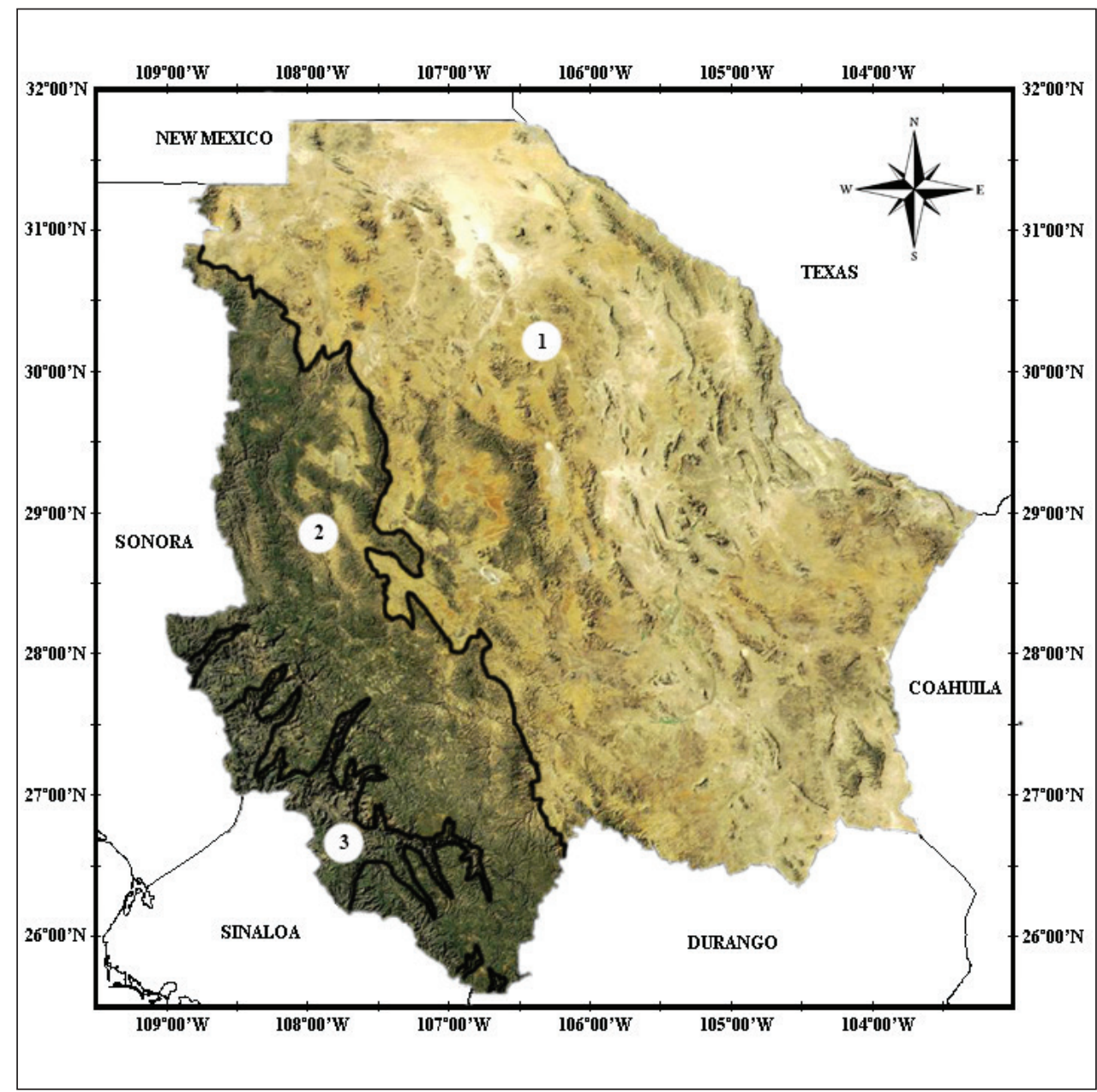

Figure I. Topographical map of the state of Chihuahua, Mexico: I Chihuahuan Desert 2 Sierra Madre Occidental, and 3 Cooper Canyon (INEGI 2001).

Among these states Chihuahua has received a great deal of attention in the study of its herpetofauna. Lemos-Espinal and H. Smith (2015a) reviewed herpetological studies previously done in this state, reporting a total of 158 publications related to amphibian and reptile species since the description of Axolotes maculata (= Ambystoma rosaceum) by Owen (1844) through the description of Incilius mccoyi by Santos-Barrera and Flores-Villela (2011), adding the recent publications by Anderson and Greenbaum (2012), Villa et al. (2012), Uriarte-Garzón and García-Vázquez (2014), Lemos-Espinal and H. Smith (2015a), and Lemos-Espinal et al. (2015). The number of publications has increased to 163. The chronological distribution of these publications is the following: prior to 1850 (1); $1851-1875$ (4); 1876-1900 (5); 1901-1925 (1); 1926-1950 (14); 1951-1975 (18); 1976-2000 (37); 2001-2015 (83), suggesting a surge in interest and knowledge about the herpetofauna of Chihuahua. 
Although there has been a considerable interest in the herpetofauna of Chihuahua, as stated above, none of these 163 publications has focused on the conservation statuses of the documented species for this state. Here, we report the list of amphibians and reptiles that have been recorded so far for the state of Chihuahua. While checklists for Chihuahua are available (e.g., Lemos-Espinal and H. Smith 2007, 2015a), we expand on these earlier efforts by also collecting and summarizing the conservation statuses for each documented species. We also compare the list of the six adjoining states in the United States and Mexico for which recent checklists are available (New Mexico, Texas, Coahuila, Durango, Sinaloa, and Sonora). Our goal is to place this checklist into a regional and conservation context not available in previously published checklists.

\section{Methods}

We compiled the list of amphibians and reptiles of the state of Chihuahua from the following sources: (1) our own field work; (2) specimens from the Laboratorio de Ecología - UBIPRO (LEUBIPRO) collections; (3) databases from the Comisión Nacional para el Conocimiento y Uso de la Biodiversidad (National Commission for the Understanding and Use of Biodiversity; CONABIO), including records from the following 22 collections Colección Herpetológica, Departamento de Zoología, Escuela Nacional de Ciencias Biológicas (ENCB); Colección Herpetológica, Museo de Zoología "Alfonso L. Herrera", Facultad de Ciencias UNAM (MZFC-UNAM); Colección Nacional de Anfibios y Reptiles, Instituto de Biología UNAM (CNAR); Amphibians and Reptiles Collection, University of Arizona (UAZ); Collection of Herpetology, Amphibians and Reptiles Section, Carnegie Museum of Natural History, Pittsburgh; Collection of Herpetology, Biology Department, Tulane University, New Orleans (TU); Collection of Herpetology, Department of Vertebrate Zoology, National Museum of Natural History, Smithsonian Institution (USNM); Collection of Herpetology, Herpetology Department, American Museum of Natural History (AMNH); Collection of Herpetology, Herpetology Department, California Academy of Sciences (CAS); Collection of Herpetology, Museum of Comparative Zoology, Harvard University Cambridge (MCZ); Collection of Herpetology, Museum of Vertebrate Zoology, Division of Biological Sciences, University of California Berkeley (MVZ); Collection of Herpetology, Museum of Zoology, University of Michigan Ann Arbor (UMMZ); Collection of Herpetology, Texas Cooperative Wildlife Collection, Texas A\&M University (TCWC); Collection of Herpetology, Texas Natural History Collection, University of Texas Austin (TNHC); Collection of Herpetology, University of Colorado Museum (UCM); Collection of Herpetology, University of Illinois Museum of Natural History (UIMNH); Division of Amphibians and Reptiles, Field Museum of Natural History (FMNH); Fort Worth Museum of Sciences and History (FWMSH); Herpetology Section, Natural History Museum of Los Angeles County (LACM); Louisiana State University, Museum of Life Sciences; Merriam Museum, University of Texas Arlington (UTAMM); Museum of Natural History, Division of 
Herpetology, Kansas University (MNHUK); and (4) a thorough examination of the available literature on amphibians and reptiles in the state. Species were included in the checklist only if we were able to confirm the record, either by direct observation or through documented museum records or vouchers in the state. In addition, we recorded the conservation status of each species based on three sources: 1) the IUCN Red List, 2) Environmental Viability Scores from Wilson et al. (2013a,b), and 3) listing in SEMARNAT (2010).

Scientific names used in this publication are based on the taxonomic list published in Lemos-Espinal (2015). The amphibian names follows Frost (2016) and the reptile names follows Uetz and Hošek (2016). State lists used to compare the species composition between Chihuahua and the adjoining states were: Dixon (2015) for Texas; Enderson et al. (2009) for Sinaloa; Lemos-Espinal and G. Smith (2016) for Coahuila; Painter and Stuart (2015) for New Mexico; Rorabaugh and Lemos-Espinal (2016) for Sonora; and Valdez-Lares et al. (2013) for Durango. We updated these lists for Coahuila (adding Crotalus ornatus, Nevárez De los Reyes et al. [2016]); Sonora and Sinaloa (adding Gopherus evgoodei, Edwards et al. [2016]); Texas (adding Crotalus ornatus, Anderson and Greenbaum [2012]); Durango (we regarded the population of Barisia imbricata [Wiegmann] as Barisia ciliaris [Smith]; Sceloporus edbelli Smith et al. as part of Sceloporus consobrinus Baird \& Girard; Sceloporus lineolateralis Smith as part of Sceloporus jarrovii Cope; and Aspidoscelis scalaris [Baird \& Girard] as part of Aspidoscelis gularis [Baird \& Girard]). We also determined the number of overlapping species between each of these states and Chihuahua.

\section{Results and discussion}

A total of 175 (173 native, two introduced) species of amphibians and reptiles is found in Chihuahua. Thirty-eight of these species are amphibians (four salamanders, 34 anurans [one introduced]), and 137 are reptiles (13 turtles, 51 lizards [one introduced], and 73 snakes) (Tables 1,2). These represent 32 families: nine amphibians (two salamanders; seven anurans), and 23 reptiles (five of turtles, 11 of lizards and seven of snakes), and 81 genera: 16 amphibians (two salamanders, 14 anurans), and 65 reptiles (seven of turtles, 20 of lizards and 38 of snakes). The introduced species are the American Bullfrog (Lithobates catesbeianus) and the Mediterranean House Gecko (Hemidactylus turcicus).

\section{General distribution}

Thirteen of the 38 species of amphibians that inhabit Chihuahua are endemic to Mexico, one of them (Lithobates lemosespinali) is restricted to a small area in the Sierra Madre Occidental of Chihuahua, and another (Isthmura sierraoccidentalis) is found only in an isolated population in the Sierra Madre Occidental of Sonora and Chihuahua. Eleven 
Table I. Checklist of amphibians and reptiles of Chihuahua providing the habitat type $(\mathrm{CD}=$ Chihuahuan Desert, SMO = Temperate Forests of the Sierra Madre Occidental, SBT = Subtropics - Canyons of the Sierra Madre Occidental; GEN = Generalist - occupies more than one habitat type), IUCN Status $(\mathrm{DD}=$ Data Deficient LC = Least Concern, $\mathrm{V}=$ Vulnerable, $\mathrm{NT}=$ Neat Threatened; $\mathrm{E}=$ Endangered; $\mathrm{CE}=$ Critically Endangered) according to the IUCN Red List (The IUCN Red List of Threatened Species, Version 2016.1; www.iucnredlist.org; accessed 30 June 2016), Environmental Vulnerability Score (EVS; the higher the score the greater the vulnerability; NE = not evaluated) from Wilson et al. (2013a,b) and Johnson et al. (2015a), and conservation status in Mexico according to SEMARNAT (2010) (P = in danger of extinction, $\mathrm{A}=$ threatened; $\mathrm{Pr}=$ subject to special protection, $\mathrm{NL}-$ not listed). Source denotes whether the species was observed in the field by the authors (A), documented in the CONABIO data base and/or museum collections (C/M), or found in the literature (citation of source).

\begin{tabular}{|c|c|c|c|c|c|}
\hline & $\begin{array}{c}\text { Habitat } \\
\text { type }\end{array}$ & IUCN & EVS & SEMARNAT & Source \\
\hline \multicolumn{6}{|l|}{ CLASS AMPHIBIA } \\
\hline \multicolumn{6}{|l|}{ ORDER CAUDATA } \\
\hline \multicolumn{6}{|l|}{ Ambystomatidae } \\
\hline Ambystoma mavortium Baird & $\mathrm{CD}$ & $\mathrm{LC}$ & 10 & NL & A \\
\hline Ambystoma rosaceum Taylor & SMO & LC & 14 & $\operatorname{Pr}$ & $\mathrm{A}$ \\
\hline Ambystoma silvense Webb & SMO & $\mathrm{DD}$ & 14 & NL & $\mathrm{C} / \mathrm{M}$ \\
\hline \multicolumn{6}{|l|}{ Plethodontidae } \\
\hline Isthmura sierraoccidentalis (Gray) & SMO & $\mathrm{V}$ & 12 & $\mathrm{~A}^{1}$ & $\mathrm{C} / \mathrm{M}$ \\
\hline
\end{tabular}

\begin{tabular}{|c|c|c|c|c|c|}
\hline \multicolumn{6}{|l|}{ Bufonidae } \\
\hline Anaxyrus cognatus (Say) & CD & LC & 8 & NL & A \\
\hline Anaxyrus debilis (Girard) & $\mathrm{CD}$ & LC & 7 & $\operatorname{Pr}$ & $\mathrm{A}$ \\
\hline Anaxyrus mexicanus (Brocchi) & SMO & NT & 13 & NL & A \\
\hline Anaxyrus punctatus (Baird \& Girard) & GEN & LC & 5 & NL & A \\
\hline Anaxyrus speciosus (Girard) & $\mathrm{CD}$ & LC & 12 & NL & $\mathrm{A}$ \\
\hline Anaxyrus woodhousii (Girard) & GEN & LC & 10 & NL & A \\
\hline Incilius alvarius (Girard) & $\mathrm{CD}$ & LC & 11 & NL & $\begin{array}{c}\text { Santos-Barrera et al. } \\
(2006)\end{array}$ \\
\hline Incilius mazatlanensis (Taylor) & SBT & LC & 12 & NL & $\mathrm{A}$ \\
\hline Incilius mccoyi Santos-Barrera \& Flores-Villela & SMO & NL & 14 & NL & A \\
\hline Rhinella horribilis (Linnaeus) & SBT & LC & 3 & NL & A \\
\hline \multicolumn{6}{|l|}{ Craugastoridae } \\
\hline Craugastor augusti (Dugès) & SBT & LC & 8 & NL & $\mathrm{C} / \mathrm{M}$ \\
\hline Craugastor tarahumaraensis (Taylor) & SMO & $\mathrm{V}$ & 17 & $\operatorname{Pr}$ & A \\
\hline \multicolumn{6}{|l|}{ Eleutherodactylidae } \\
\hline $\begin{array}{l}\text { Eleutherodactylus interorbitalis (Langebartel \& } \\
\text { Shannon) }\end{array}$ & SBT & $\mathrm{DD}$ & 15 & $\operatorname{Pr}$ & A \\
\hline Eleutherodactylus marnockii (Cope) & $\mathrm{CD}$ & LC & $\mathrm{NE}$ & NL & A \\
\hline \multicolumn{6}{|l|}{ Hylidae } \\
\hline Hyla arenicolor Cope & SMO & LC & 7 & NL & A \\
\hline Hyla wrightorum Taylor, 1939 & SMO & LC & 9 & NL & $\mathrm{A}$ \\
\hline Agalychnis dacnicolor (Cope) & SBT & LC & 13 & NL & A \\
\hline Smilisca baudinii (Duméril \& Bibron) & SBT & LC & 3 & NL & A \\
\hline Tlalocohyla smithii (Boulenger) & SBT & LC & 11 & NL & A \\
\hline
\end{tabular}




\begin{tabular}{|c|c|c|c|c|c|}
\hline & $\begin{array}{c}\text { Habitat } \\
\text { type }\end{array}$ & IUCN & EVS & SEMARNAT & Source \\
\hline \multicolumn{6}{|l|}{ Microhylidae } \\
\hline Gastrophryne mazatlanensis (Taylor) & SBT & NL & 8 & NL & A \\
\hline Gastrophryne olivacea (Hallowell) & $\mathrm{CD}$ & $\mathrm{LC}$ & 9 & $\operatorname{Pr}$ & A \\
\hline Hypopachus variolosus (Cope) & SBT & LC & 4 & NL & $\mathrm{A}$ \\
\hline \multicolumn{6}{|l|}{ Ranidae } \\
\hline Lithobates berlandieri (Baird) & $\mathrm{CD}$ & LC & 7 & $\operatorname{Pr}$ & $\mathrm{A}$ \\
\hline Lithobates catesbeianus (Shaw) - Introduced & SMO & $\mathrm{LC}$ & 10 & NL & $\mathrm{A}$ \\
\hline Lithobates chiricahuensis (Platz \& Mecham) & SMO & $\mathrm{V}$ & 11 & A & $\mathrm{A}$ \\
\hline Lithobates forreri (Boulenger) & SBT & LC & 3 & $\operatorname{Pr}$ & $\mathrm{A}$ \\
\hline $\begin{array}{l}\text { Lithobates lemosespinali (Smith \& Chiszar) } \\
\text { Endemic }\end{array}$ & SMO & $\mathrm{DD}$ & 14 & NL & A \\
\hline Lithobates magnaocularis (Frost \& Bagnara) & GEN & LC & 12 & NL & $\mathrm{A}$ \\
\hline Lithobates pustulosus (Boulenger) & SBT & LC & 9 & $\operatorname{Pr}$ & $\mathrm{C} / \mathrm{M}$ \\
\hline Lithobates tarahumarae (Boulenger) & SMO & $\mathrm{V}$ & 8 & NL & A \\
\hline Lithobates yavapaiensis (Platz \& Frost) & SMO & LC & 12 & $\operatorname{Pr}$ & $\mathrm{A}$ \\
\hline \multicolumn{6}{|l|}{ Scaphiopodidae } \\
\hline Scaphiopus couchi Baird & GEN & LC & 3 & NL & $\mathrm{A}$ \\
\hline Spea bombifrons (Cope) & $\mathrm{CD}$ & LC & 10 & NL & $\mathrm{A}$ \\
\hline Spea multiplicata (Cope) & GEN & LC & 6 & NL & A \\
\hline
\end{tabular}
ORDER TESTUDINES

\section{Emydidae}

\begin{tabular}{|c|c|c|c|c|c|}
\hline Chrysemys picta (Schneider) & GEN & LC & 14 & A & A \\
\hline Terrapene nelsoni Stejneger & SMO & DD & 18 & $\operatorname{Pr}$ & A \\
\hline Terrapene ornata (Agassiz) & $\mathrm{CD}$ & NT & 15 & $\operatorname{Pr}$ & A \\
\hline Trachemys gaigeae (Hartweg) & $\mathrm{CD}$ & $\mathrm{V}$ & 18 & $\mathrm{NL}$ & A \\
\hline \multicolumn{6}{|l|}{ Geoemydidae } \\
\hline Rhinoclemmys pulcherrima (Gray) & SBT & NL & 8 & NL & A \\
\hline \multicolumn{6}{|l|}{ Kinosternidae } \\
\hline Kinosternon durangoense Iverson & $\mathrm{CD}$ & DD & 16 & $\mathrm{NL}$ & A \\
\hline Kinosternon flavescens (Agassiz) & $\mathrm{CD}$ & $\mathrm{LC}$ & 12 & NL & A \\
\hline Kinosternon hirtipes (Wagler) & GEN & LC & 10 & $\operatorname{Pr}$ & A \\
\hline Kinosternon integrum LeConte & SBT & $\mathrm{LC}$ & 11 & $\operatorname{Pr}$ & A \\
\hline Kinosternon sonoriense Le Conte & GEN & NT & 14 & $\begin{array}{c}\mathrm{P}-\text { subsp } \\
\text { longifemorale }\end{array}$ & A \\
\hline \multicolumn{6}{|l|}{ Testudinidae } \\
\hline Gopherus flavomarginatus Legler & $\mathrm{CD}$ & $\mathrm{V}$ & 19 & $\mathrm{P}$ & A \\
\hline $\begin{array}{l}\text { Gopherus evgoodei Edwards, Karl, Vaughn, } \\
\text { Rosen, Meléndez-Torres, \& Murphy }\end{array}$ & SBT & NL & $\mathrm{NE}$ & $\mathrm{A}^{2}$ & A \\
\hline \multicolumn{6}{|l|}{ Trionychidae } \\
\hline Apalone spinifera (Le Sueur) & $\mathrm{CD}$ & LC & 15 & $\operatorname{Pr}$ & A \\
\hline \multicolumn{6}{|l|}{ ORDER SQUAMATA } \\
\hline \multicolumn{6}{|l|}{ SUBORDER LACERTILIA } \\
\hline \multicolumn{6}{|l|}{ Anguidae } \\
\hline Barisia ciliaris (Smith) & SMO & NL & 15 & $\mathrm{NL}$ & A \\
\hline Barisia levicollis Stejneger Endemic & SMO & DD & 15 & $\operatorname{Pr}$ & A \\
\hline
\end{tabular}




\begin{tabular}{|c|c|c|c|c|c|}
\hline & \begin{tabular}{|c|}
$\begin{array}{c}\text { Habitat } \\
\text { type }\end{array}$ \\
\end{tabular} & IUCN & EVS & SEMARNAT & Source \\
\hline Elgaria kingii Gray & SMO & LC & 10 & $\operatorname{Pr}$ & $\mathrm{A}$ \\
\hline Gerrhonotus infernalis Baird & SMO & LC & 13 & NL & $\mathrm{A}$ \\
\hline \multicolumn{6}{|l|}{ Crotaphytidae } \\
\hline Crotaphytus collaris (Say) & GEN & LC & 13 & A & $\mathrm{A}$ \\
\hline Gambelia wislizenii (Baird \& Girard) & $\mathrm{CD}$ & LC & 13 & $\operatorname{Pr}$ & A \\
\hline \multicolumn{6}{|l|}{ Dactyloidae } \\
\hline Anolis nebulosus (Wiegmann) & SBT & LC & 13 & NL & A \\
\hline \multicolumn{6}{|l|}{ Eublepharidae } \\
\hline Coleonyx brevis Stejneger & $\mathrm{CD}$ & LC & 14 & $\operatorname{Pr}$ & A \\
\hline \multicolumn{6}{|l|}{ Gekkonidae (INTRODUCED) } \\
\hline Hemidactylus turcicus (Linnaeus) Introduced & & N/A & N/A & N/A & A \\
\hline \multicolumn{6}{|l|}{ Helodermatidae } \\
\hline Heloderma horridum Wiegmann & SBT & LC & 11 & $\mathrm{~A}$ & A \\
\hline \multicolumn{6}{|l|}{ Iguanidae } \\
\hline Ctenosaura macrolopha Smith & SBT & NL & 19 & $\operatorname{Pr}^{1}$ & A \\
\hline \multicolumn{6}{|l|}{ Phrynosomatidae } \\
\hline Cophosaurus texanus Troschel & $\mathrm{CD}$ & LC & 14 & A & A \\
\hline Holbrookia approximans Baird & $\mathrm{CD}$ & NL & 14 & NL & A \\
\hline Holbrookia elegans Bocourt & GEN & LC & 13 & NL & A \\
\hline Holbrookia maculata Girard & GEN & LC & 10 & NL & $\mathrm{A}$ \\
\hline Phrynosoma cornutum (Harlan) & $\mathrm{CD}$ & LC & 11 & NL & A \\
\hline Phrynosoma hernandesi Girard & SMO & LC & 13 & NL & A \\
\hline Phrynosoma modestum Girard & $\mathrm{CD}$ & LC & 12 & NL & A \\
\hline Phrynosoma orbiculare (Linnaeus) & SMO & LC & 12 & A & A \\
\hline Sceloporus albiventris Smith & SBT & NL & 16 & NL & A \\
\hline Sceloporus bimaculosus Phelan \& Brattstrom & $\mathrm{CD}$ & NL & $\mathrm{NE}$ & NL & A \\
\hline Sceloporus clarkii Baird \& Girard & GEN & $\mathrm{LC}$ & 10 & NL & A \\
\hline Sceloporus consobrinus Baird \& Girard & $\mathrm{CD}$ & NL & $\mathrm{NE}$ & NL & A \\
\hline Sceloporus cowlesi Lowe \& Norris & $\mathrm{CD}$ & NL & 13 & NL & $\mathrm{A}$ \\
\hline Sceloporus jarrovii Cope & SMO & LC & 11 & NL & $\mathrm{A}$ \\
\hline Sceloporus lemosespinali Lara-Góngora & SMO & DD & 16 & NL & $\mathrm{A}$ \\
\hline Sceloporus merriami Stejneger & $\mathrm{CD}$ & LC & 13 & NL & $\mathrm{A}$ \\
\hline Sceloporus nelsoni Cochran & SBT & LC & 13 & NL & A \\
\hline Sceloporus poinsettii Baird \& Girard & $\mathrm{CD}$ & LC & 12 & NL & A \\
\hline Sceloporus slevini Smith & SMO & $\mathrm{LC}$ & 11 & NL & A \\
\hline Sceloporus virgatus Smith & SMO & LC & 15 & NL & A \\
\hline $\begin{array}{l}\text { Uma paraphygas Williams, Chrapliwy \& } \\
\text { Smith }\end{array}$ & $\mathrm{CD}$ & NT & 17 & $\mathrm{P}$ & A \\
\hline Urosaurus bicarinatus (Duméril) & SBT & $\mathrm{LC}$ & 12 & NL & A \\
\hline Urosaurus ornatus (Baird \& Girard) & GEN & LC & 10 & NL & $\mathrm{A}$ \\
\hline Uta stansburiana Baird \& Girard & $\mathrm{CD}$ & LC & 11 & A & A \\
\hline \multicolumn{6}{|l|}{ Phyllodactylidae } \\
\hline Phyllodactylus tuberculosus Wiegmann & SBT & LC & 8 & NL & A \\
\hline \multicolumn{6}{|l|}{ Scincidae } \\
\hline Plestiodon bilineatus (Tanner) & SMO & NL & 13 & NL & $\mathrm{A}$ \\
\hline Plestiodon callicephalus (Bocourt) & SMO & $\mathrm{LC}$ & 12 & NL & $\mathrm{A}$ \\
\hline
\end{tabular}




\begin{tabular}{|c|c|c|c|c|c|}
\hline & $\begin{array}{c}\text { Habitat } \\
\text { type }\end{array}$ & IUCN & EVS & SEMARNAT & Source \\
\hline Plestiodon multilineatus (Tanner) Endemic & SMO & $\mathrm{DD}$ & 16 & $\operatorname{Pr}$ & $\begin{array}{c}\text { Van Devender and } \\
\text { Van Devender } \\
(1975)\end{array}$ \\
\hline Plestiodon multivirgatus (Hallowell) & $\mathrm{CD}$ & LC & 14 & $\operatorname{Pr}$ & $\mathrm{A}$ \\
\hline Plestiodon obsoletus (Baird \& Girard) & $\mathrm{CD}$ & LC & 11 & NL & A \\
\hline Plestiodon parviauriculatus (Taylor) & SMO & $\mathrm{DD}$ & 15 & $\operatorname{Pr}$ & A \\
\hline Plestiodon tetragrammus (Baird) & $\mathrm{CD}$ & $\mathrm{LC}$ & 12 & NL & A \\
\hline \multicolumn{6}{|l|}{ Teiidae } \\
\hline Aspidoscelis costata (Cope) & SBT & NL & 11 & $\operatorname{Pr}$ & A \\
\hline Aspidoscelis exsanguis (Lowe) & $\mathrm{CD}$ & LC & 14 & NL & $\mathrm{A}$ \\
\hline Aspidoscelis gularis (Baird \& Girard) & $\mathrm{CD}$ & LC & 9 & NL & A \\
\hline Aspidoscelis inornata (Baird) & $\mathrm{CD}$ & LC & 14 & NL & $\mathrm{A}$ \\
\hline Aspidoscelis marmorata (Baird \& Girard) & $\mathrm{CD}$ & NL & 14 & NL & A \\
\hline Aspidoscelis sonorae (Lowe \& Wright) & SMO & $\mathrm{LC}$ & 13 & NL & A \\
\hline Aspidoscelis tesselata (Say) & $\mathrm{CD}$ & $\mathrm{LC}$ & 14 & NL & $\mathrm{A}$ \\
\hline Aspidoscelis uniparens (Wright \& Lowe) & $\mathrm{CD}$ & $\mathrm{LC}$ & 15 & NL & A \\
\hline
\end{tabular}

\section{ORDER SQUAMATA}

\section{SUBORDER SERPENTES}

\section{Boidae}

\begin{tabular}{|c|c|c|c|c|c|}
\hline Boa sigma Daudin & SBT & NL & NE & $\mathrm{A}^{3}$ & A \\
\hline \multicolumn{6}{|l|}{ Colubridae } \\
\hline Arizona elegans Kennicott & $\mathrm{CD}$ & $\mathrm{LC}$ & 5 & NL & A \\
\hline Bogertophis subocularis (Brown) & $\mathrm{CD}$ & LC & 14 & NL & A \\
\hline Conopsis nasus Günther & $\mathrm{SMO}$ & $\mathrm{LC}$ & 11 & NL & A \\
\hline $\begin{array}{l}\text { Drymarchon melanurus (Duméril, Bibron \& } \\
\text { Duméril) }\end{array}$ & SBT & $\mathrm{LC}$ & 6 & NL & A \\
\hline Drymobius margaritiferus (Schlegel) & SBT & NL & 6 & NL & A \\
\hline Gyalopion canum Cope & $\mathrm{CD}$ & $\mathrm{LC}$ & 9 & NL & A \\
\hline Gyalopion quadrangulare (Günther) & SBT & $\mathrm{LC}$ & 11 & $\operatorname{Pr}$ & A \\
\hline Lampropeltis getula (Linnaeus) & GEN & $\mathrm{LC}$ & $\mathrm{NE}$ & A & A \\
\hline Lampropeltis knoblochi Taylor & SMO & NL & 10 & $\mathrm{~A}^{4}$ & A \\
\hline Lampropeltis polyzona Cope & SBT & NL & 11 & NL & A \\
\hline Leptophis diplotropis (Günther) & SBT & $\mathrm{LC}$ & 14 & A & A \\
\hline Masticophis bilineatus Jan & GBN & $\mathrm{LC}$ & 11 & NL & A \\
\hline Masticophis flagellum Shaw & $\mathrm{CD}$ & $\mathrm{LC}$ & 8 & A & A \\
\hline $\begin{array}{l}\text { Masticophis mentovarius (Duméril, Bibron \& } \\
\text { Duméril) }\end{array}$ & SBT & $\mathrm{LC}$ & 6 & A & A \\
\hline Masticophis taeniatus (Hallowell) & GEN & $\mathrm{LC}$ & 10 & NL & A \\
\hline Mastigodryas cliftoni (Hardy) & SBT & NL & 14 & NL & A \\
\hline Opheodrys vernalis (Harlan) & $\mathrm{SMO}$ & $\mathrm{LC}$ & NE & NL & $\begin{array}{l}\text { Van Devender and } \\
\text { Lowe (1977) }\end{array}$ \\
\hline Oxybelis aeneus (Wagler) & SBT & NL & 5 & NL & $\mathrm{A}$ \\
\hline Pantherophis emoryi (Baird \& Girard) & $\mathrm{CD}$ & $\mathrm{LC}$ & 13 & NL & A \\
\hline Pituophis catenifer (Blainville) & GEN & $\mathrm{LC}$ & 9 & NL & A \\
\hline Pituophis deppei (Duméril) & SMO & $\mathrm{LC}$ & 14 & A & A \\
\hline Rhinocheilus lecontei Baird \& Girard & $\mathrm{CD}$ & $\mathrm{LC}$ & 8 & NL & A \\
\hline Salvadora bairdii Jan \& Sordelli & SMO & $\mathrm{LC}$ & 15 & $\operatorname{Pr}$ & A \\
\hline
\end{tabular}




\begin{tabular}{|c|c|c|c|c|c|}
\hline & $\begin{array}{c}\text { Habitat } \\
\text { type }\end{array}$ & IUCN & EVS & SEMARNAT & Source \\
\hline Salvadora deserticola Schmidt & $\mathrm{CD}$ & NL & 14 & NL & A \\
\hline Salvadora grahamiae Baird \& Girard & GEN & LC & 10 & NL & $\mathrm{A}$ \\
\hline Senticolis triaspis (Cope) & SBT & $\mathrm{LC}$ & 6 & NL & $\mathrm{A}$ \\
\hline Sonora aemula (Cope) & SBT & NT & 16 & $\operatorname{Pr}$ & $\mathrm{A}$ \\
\hline Sonora semiannulata Baird \& Girard & GEN & LC & 5 & NL & $\mathrm{A}$ \\
\hline Sympholis lippiens Cope & SBT & NL & 14 & NL & $\mathrm{A}$ \\
\hline Tantilla hobartsmithi Taylor & $\mathrm{CD}$ & $\mathrm{LC}$ & 11 & NL & $\mathrm{A}$ \\
\hline Tantilla nigriceps Kennicott & $\mathrm{CD}$ & LC & 11 & NL & $\mathrm{A}$ \\
\hline Tantilla wilcoxi Stejneger & SMO & $\mathrm{LC}$ & 10 & NL & $\mathrm{A}$ \\
\hline Tantilla yaquia Smith & SBT & $\mathrm{LC}$ & 10 & NL & $\mathrm{A}$ \\
\hline Trimorphodon tau Cope & SBT & $\mathrm{LC}$ & 13 & NL & A \\
\hline Trimorphodon vilkinsonii Cope & $\mathrm{CD}$ & LC & 15 & $\mathrm{~A}$ & $\mathrm{~A}$ \\
\hline \multicolumn{6}{|l|}{ Dipsidae } \\
\hline Diadophis punctatus (Linnaeus) & GEN & $\mathrm{LC}$ & 4 & NL & $\mathrm{A}$ \\
\hline Geophis dugesii Bocourt & SMO & LC & 13 & $\mathrm{NL}$ & $\mathrm{A}$ \\
\hline Heterodon kennerlyi Kennicott & $\mathrm{CD}$ & NL & 11 & $\operatorname{Pr}$ & $\mathrm{A}$ \\
\hline Hypsiglena chlorophaea Cope & GEN & NL & 8 & NL & $\mathrm{A}$ \\
\hline Hypsiglena jani (Dugès) & $\mathrm{CD}$ & NL & 6 & NL & $\mathrm{A}$ \\
\hline Imantodes gemmistratus (Cope) & SBT & NL & 6 & $\operatorname{Pr}$ & $\mathrm{A}$ \\
\hline Leptodeira splendida (Günther) & SBT & LC & 14 & NL & $\mathrm{A}$ \\
\hline Rhadinaea hesperia Bailey & SMO & $\mathrm{LC}$ & 10 & $\begin{array}{c}\text { Pr - subsp } \\
\text { baileyi }\end{array}$ & A \\
\hline Rhadinaea laureata (Günther) & SMO & $\mathrm{LC}$ & 12 & $\mathrm{NL}$ & Villa et al. (2012) \\
\hline $\begin{array}{l}\text { Tropidodipsas repleta Smith, Lemos-Espinal, } \\
\text { Hartman \& Chiszar }\end{array}$ & SBT & $\mathrm{DD}$ & 17 & NL & A \\
\hline \multicolumn{6}{|l|}{ Elapidae } \\
\hline Micruroides euryxanthus (Kennicott) & SON & NL & 15 & A & A \\
\hline Micrurus distans (Kennicott) & SBT & LC & 14 & $\operatorname{Pr}$ & $\mathrm{A}$ \\
\hline \multicolumn{6}{|l|}{ Leptotyphlopidae } \\
\hline Rena dissecta (Cope) & $\mathrm{CD}$ & $\mathrm{LC}$ & 11 & NL & $\mathrm{C} / \mathrm{M}$ \\
\hline Rena humilis Baird \& Girard & $\mathrm{CD}$ & LC & 8 & NL & $\mathrm{A}$ \\
\hline Rena segrega (Klauber) & $\mathrm{CD}$ & NL & $\mathrm{NE}$ & NL & $\mathrm{C} / \mathrm{M}$ \\
\hline \multicolumn{6}{|l|}{ Natricidae } \\
\hline Nerodia erythrogaster (Forster) & $\mathrm{CD}$ & $\mathrm{LC}$ & 11 & A & $\begin{array}{l}\text { Uriarte-Garzón and } \\
\text { García-Vázquez } \\
(2014)\end{array}$ \\
\hline Storeria storerioides (Cope) & SMO & LC & 11 & NL & $\mathrm{A}$ \\
\hline Thamnophis cyrtopsis (Kennicott) & GEN & LC & 7 & A & $\mathrm{A}$ \\
\hline Thamnophis elegans (Baird \& Girard) & SMO & $\mathrm{LC}$ & 14 & $\mathrm{~A}$ & $\mathrm{~A}$ \\
\hline Thamnophis eques (Reuss) & GEN & LC & 8 & $\mathrm{~A}$ & $\mathrm{~A}$ \\
\hline Thamnophis errans Smith & SMO & LC & 16 & NL & $\mathrm{A}$ \\
\hline Thamnophis marcianus (Baird \& Girard) & GEN & LC & 10 & $\mathrm{~A}$ & $\mathrm{~A}$ \\
\hline Thamnophis melanogaster (Peters) & SMO & $\mathrm{E}$ & 15 & A & A \\
\hline Thamnophis sirtalis (Linnaeus) & SMO & LC & 14 & $\operatorname{Pr}$ & $\mathrm{A}$ \\
\hline Thamnophis unilabialis Tanner & SMO & NL & $\mathrm{NE}$ & NL & $\mathrm{A}$ \\
\hline Thamnophis validus (Kennicott) & SBT & NL & 12 & NL & $\mathrm{A}$ \\
\hline
\end{tabular}




\begin{tabular}{l|c|c|c|c|c|c}
\hline & $\begin{array}{c}\text { Habitat } \\
\text { type }\end{array}$ & IUCN & EVS & SEMARNAT & Source \\
\hline Viperidae & SBT & NT & 11 & $\operatorname{Pr}$ & $\mathrm{A}$ \\
\hline Agkistrodon bilineatus (Günther) & CD & LC & 14 & NL & C/M \\
\hline Agkistrodon contortrix (Linnaeus) & CD & LC & 9 & $\operatorname{Pr}$ & $\mathrm{A}$ \\
\hline Crotalus atrox Baird \& Girard & SBT & LC & 16 & $\operatorname{Pr}$ & $\mathrm{A}$ \\
\hline Crotalus basiliscus (Cope) & SMO & LC & 12 & $\operatorname{Pr}$ & $\mathrm{A}$ \\
\hline Crotalus lepidus (Kennicott) & GEN & LC & 8 & $\operatorname{Pr}$ & $\mathrm{A}$ \\
\hline Crotalus molossus Baird \& Girard & CD & NL & 13 & NL & $\begin{array}{c}\text { Anderson and } \\
\text { Greenbaum (2012) }\end{array}$ \\
\hline Crotalus ornatus Hallowell & SMO & LC & 14 & $\operatorname{Pr}$ & $\mathrm{A}$ \\
\hline Crotalus pricei Van Denburgh & CD & LC & 11 & $\operatorname{Pr}$ & $\mathrm{A}$ \\
\hline Crotalus scutulatus (Kennicott) & CD & LC & 12 & $\operatorname{Pr}$ & $\mathrm{A}$ \\
\hline Crotalus viridis (Rafinesque) & SMO & LC & 13 & $\operatorname{Pr}$ & $\mathrm{A}$ \\
\hline Crotalus willardi Meek &
\end{tabular}

$\mathrm{A}^{1}=$ Protected under the name Pseudoeurycea bellii; $\mathrm{A}^{2}=$ Protected under the name Gopherus agassizii; $\mathrm{A}^{3}=$ Protected under the name Boa constrictor; $\mathrm{A}^{4}=$ Protected under the name Lampropeltis pyromelana; $\operatorname{Pr}^{1}=$ Protected under the name Ctenosaura hemilopha.

more are distributed in western Mexico (Ambystoma rosaceum, A. silvense, Anaxyrus mexicanus, Incilius mazatlanensis, I. mccoyi, Crangastor tarahumaraensis [Fig. 2], Eleutherodactylus interorbitalis, Agalychnis dacnicolor, Tlalocohyla smithii, Lithobates magnaocularis, and L. pustulosus). Three more species are widely distributed from southern Canada to northern Mexico (Ambystoma mavortium, Anaxyrus cognatus, and Spea bombifrons). One species (Lithobates forreri) is widely distributed from northern Mexico to Central America, with a range that extends from central western Sonora through the Pacific Coast to Costa Rica. Another sixteen species occur from central or southern United States to northern, central or southern Mexico (Anaxyrus debilis, A. punctatus, A. speciosus, A. woodhousii, Incilius alvarius, Crangastor angusti, Eleutherodactylus marnockii, Hyla arenicolor, H. wrightorum, Gastrophryne mazatlanensis, G. olivacea, Lithobates chiricahuensis, L. tarahumarae, L. yavapaiensis, Scaphiopus couchii, and Spea multiplicata). Four more occur from eastern and/or southeastern United States to South America (Rhinella horribilis, Smilisca baudinii, Hypopachus variolosus, and Lithobates berlandieri), this last species with isolated populations in the Sierra Madre Occidental. Only one of the amphibian species that currently inhabit Chihuahua was introduced to the state (Lithobates catesbeianus).

Five of the 13 species of turtles that inhabit Chihuahua are endemic to Mexico, two of them to the Bolsón de Mapimí, a small area in southeastern Chihuahua, southwestern Coahuila, and northeastern Durango (Kinosternon durangoense and Gopherus flavomarginatus), two more to western Mexico (Terrapene nelsoni and Kinosternon integrum), and one more to the subtropics of southeastern Sonora, southwestern Chihuahua, and northern Sinaloa (Gopherus evgoodei). Six more species occur from central or southern United States to northern (Terrapene ornata [Fig. 3], Trachemys gaigeae, Kinosternon flavescens, and K. sonoriense) or central or southern Mexico (Kinosternon hirtipes 
Table 2. Summary of species present in Chihuahua by family, order or suborder, and class. Status summary indicates the number of species found in each IUCN conservation status in the Order DD, LC, V, NT, E, CE (see Table 1 for abbreviations; in some cases species have not been assigned a status by the IUCN and therefore these may not add up to the total number of species in a taxon). Mean EVS is the mean Environmental Vulnerability Score, scores $\geq 14$ are considered high vulnerability (Wilson et al. 2013a,b) and conservation status in Mexico according to SEMARNAT (2010) in the Order NL, Pr, A, P (see Table 1 for abbreviations).

\begin{tabular}{|c|c|c|c|c|c|c|c|}
\hline Class & $\begin{array}{c}\text { Order/ } \\
\text { Suborder }\end{array}$ & Family & Genera & Species & $\begin{array}{c}\text { Status } \\
\text { Summary }\end{array}$ & $\begin{array}{c}\text { Mean } \\
\text { EVS }\end{array}$ & SEMARNAT \\
\hline \multirow[t]{12}{*}{ Amphibia } & Caudata & & 2 & 4 & $1,2,1,0,0,0$ & 12.5 & $2,1,1,0$ \\
\hline & & Ambystomatidae & 1 & 3 & $1,2,0,0,0,0$ & 12.7 & $2,1,0,0$ \\
\hline & & Plethodontidae & 1 & 1 & $0,0,1,0,0,0$ & 12 & $0,0,1,0$ \\
\hline & Anura & & 14 & 34 & $2,26,3,1,0,0$ & 9.8 & $25,8,1,0$ \\
\hline & & Bufonidae & 3 & 10 & $0,8,0,1,0,0$ & 9.5 & $9,1,0,0$ \\
\hline & & Craugastoridae & 1 & 2 & $0,1,1,0,0,0$ & 12.5 & $1,1,0,0$ \\
\hline & & Eleutherodactylidae & 1 & 2 & $1,1,0,0,0,0$ & 15 & $1,1,0,0$ \\
\hline & & Hylidae & 4 & 5 & $0,5,0,0,0,0$ & 8.6 & $5,0,0,0$ \\
\hline & & Microhylidae & 2 & 3 & $0,2,0,0,0,0$ & 7 & $2,1,0,0$ \\
\hline & & Ranidae & 1 & 9 & $1,6,2,0,0,0$ & 9.6 & $4,4,1,0$ \\
\hline & & Scaphiopodidae & 2 & 3 & $0,3,0,0,0,0$ & 6.3 & $3,0,0,0$ \\
\hline & Subtotal & & 16 & 38 & $3,28,4,1,0,0$ & 9.96 & $27,9,2,0$ \\
\hline \multirow[t]{28}{*}{ Reptilia } & Testudines & & 7 & 13 & $2,5,2,2,0,0$ & 14.16 & $5,6,2,0$ \\
\hline & & Emydidae & 3 & 4 & $1,1,1,1,0,0$ & 16.25 & $1,2,1,0$ \\
\hline & & Geoemydidae & 1 & 1 & $0,0,0,0,0,0$ & 8 & $1,0,0,0$ \\
\hline & & Kinosternidae & 1 & 5 & $1,3,0,1,0,0$ & 12.6 & $3,2,0,0$ \\
\hline & & Testudinidae & 1 & 2 & $0,0,1,0,0,0$ & 19 & $0,1,1,0$ \\
\hline & & Trionychidae & 1 & 1 & $0,1,0,0,0,0$ & 15 & $0,1,0,0$ \\
\hline & Squamata & & & & & & \\
\hline & Lacertilia & & 20 & 51 & $4,34,0,1,0,0$ & 12.91 & $35,9,5,1$ \\
\hline & & Anguidae & 3 & 4 & $1,2,0,0,0,0$ & 13.25 & $2,2,0,0$ \\
\hline & & Crotaphytidae & 2 & 2 & $0,2,0,0,0,0$ & 13 & $0,1,1,0$ \\
\hline & & Dactyloidae & 1 & 1 & $0,1,0,0,0,0$ & 13 & $1,0,0,0$ \\
\hline & & Eublepharidae & 1 & 1 & $0,1,0,0,0,0$ & 14 & $0,1,0,0$ \\
\hline & & Gekkonidae & 1 & 1 & & & \\
\hline & & Helodermatidae & 1 & 1 & $0,1,0,0,0,0$ & 11 & $0,0,1,0$ \\
\hline & & Iguanidae & 1 & 1 & $0,0,0,0,0,0$ & 19 & $0,1,0,0$ \\
\hline & & Phrynosomatidae & 7 & 24 & $1,16,0,1,0,0$ & 12.7 & $20,0,3,1$ \\
\hline & & Phyllodactylidae & 1 & 1 & $0,1,0,0,0,0$ & 8 & $1,0,0,0$ \\
\hline & & Scincidae & 1 & 7 & $2,4,0,0,0,0$ & 13.3 & $4,3,0,0$ \\
\hline & & Teiidae & 1 & 8 & $0,6,0,0,0,0$ & 13 & $7,1,0,0$ \\
\hline & Serpentes & & 38 & 73 & $1,52,0,2,1,0$ & 10.95 & $38,17,16,0$ \\
\hline & & Boidae & 1 & 1 & $0,0,0,0,0,0$ & $?$ & $0,0,1,0$ \\
\hline & & Colubridae & 21 & 35 & $0,27,0,1,0,0$ & 10.5 & $23,3,7,0$ \\
\hline & & Dipsidae & 8 & 10 & $1,5,0,0,0,0$ & 10.1 & $7,3,0,0$ \\
\hline & & Elapidae & 2 & 2 & $0,1,0,0,0,0$ & 14.5 & $0,1,1,0$ \\
\hline & & Leptotyphlopidae & 1 & 3 & $0,2,0,0,0,0$ & 9.5 & $3,0,0,0$ \\
\hline & & Natricidae & 3 & 11 & $0,8,0,0,1,0$ & 11.8 & $4,1,6.0$ \\
\hline & & Viperidae & 2 & 11 & $0,9,0,1,0,0$ & 12.1 & $1,9,0,0$ \\
\hline & Subtotal & & 65 & 137 & $7,91,2,5,1,0$ & 11.99 & $78,32,23,1$ \\
\hline TOTAL & & & 81 & 175 & $10,119,6,6,1,0$ & 11.45 & $105,41,25,1$ \\
\hline
\end{tabular}




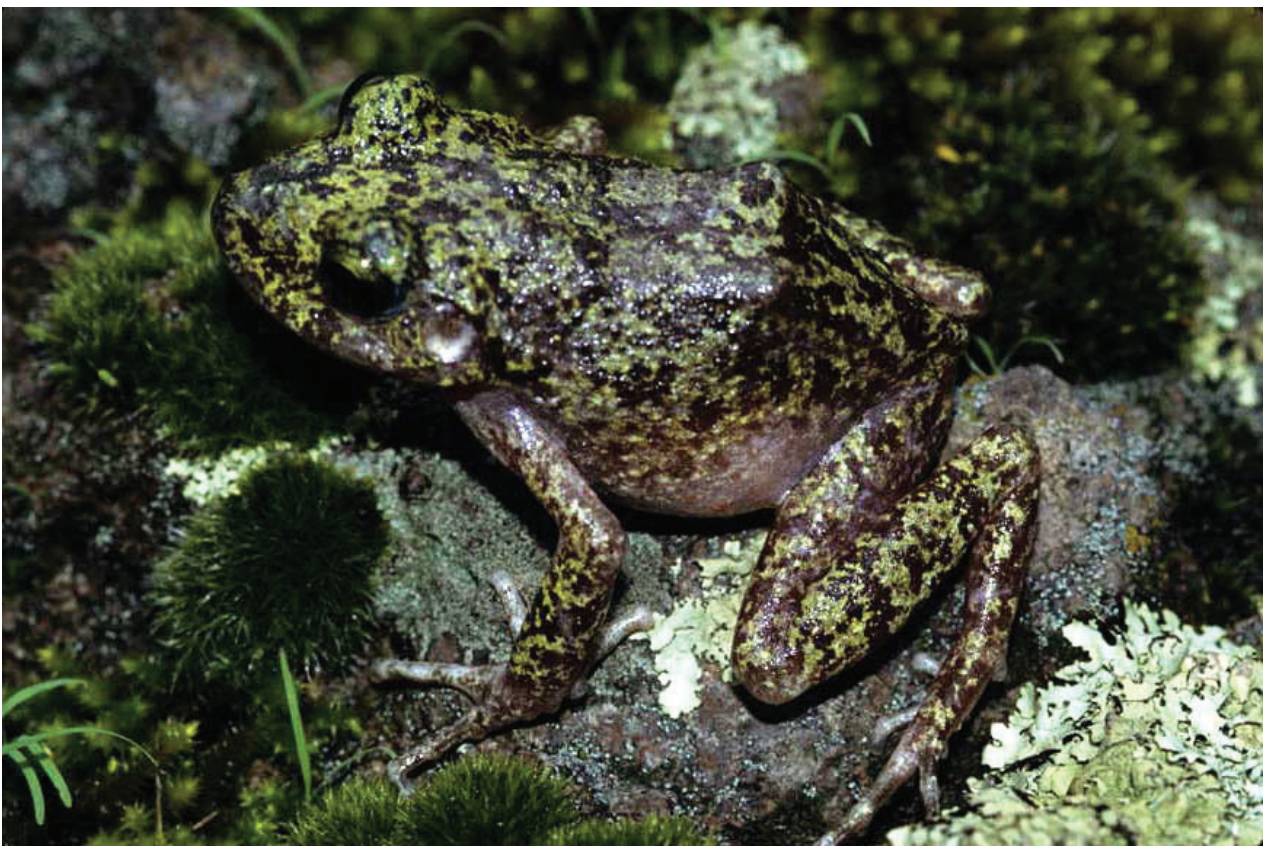

Figure 2. Crangastor tarahumaraensis. Ocampo, Chihuahua. Photo courtesy of Peter Heimes.

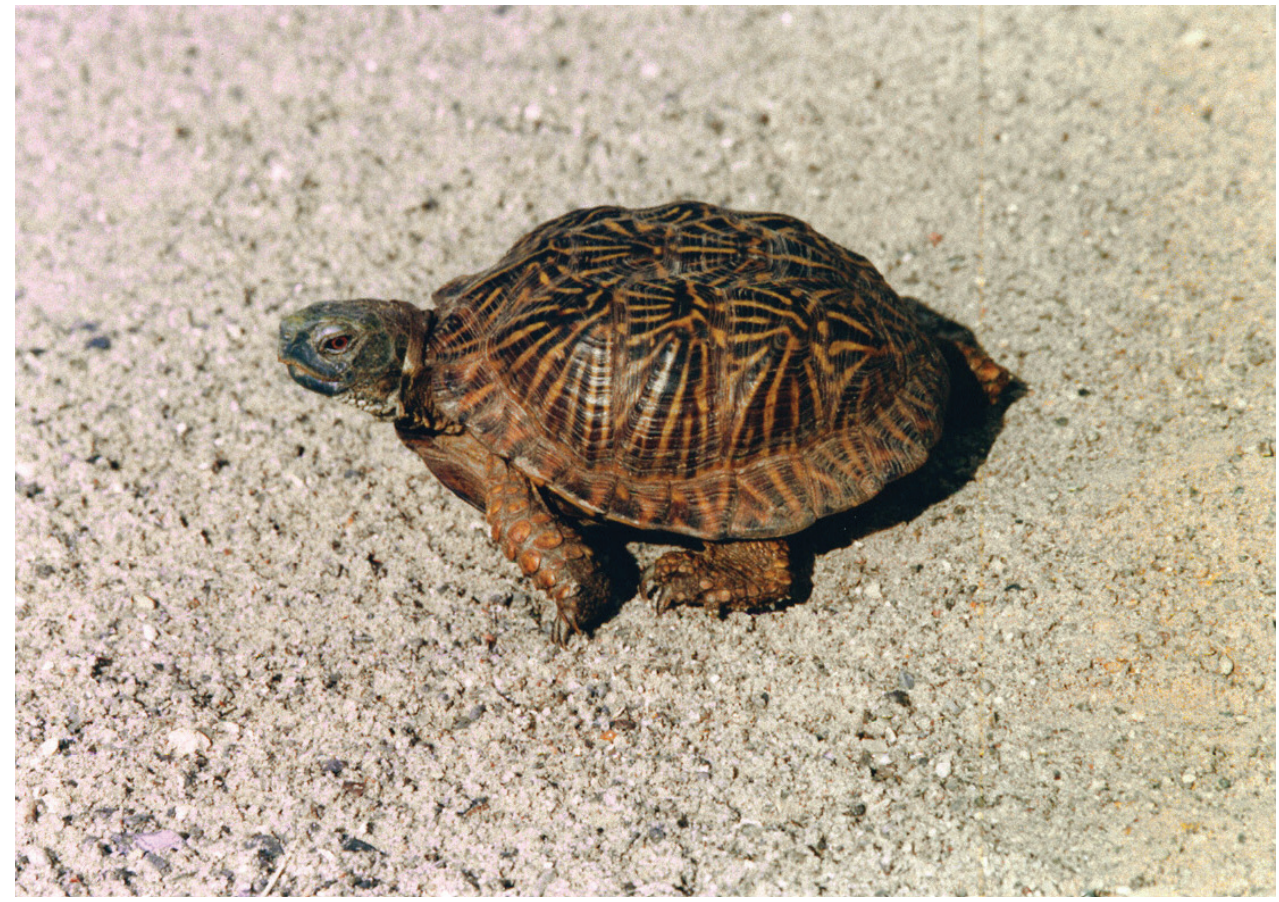

Figure 3. Terrapene ornata. Rancho de Flores Magón, Buenaventura, Chihuahua. Photo by Julio Lemos Espinal. 


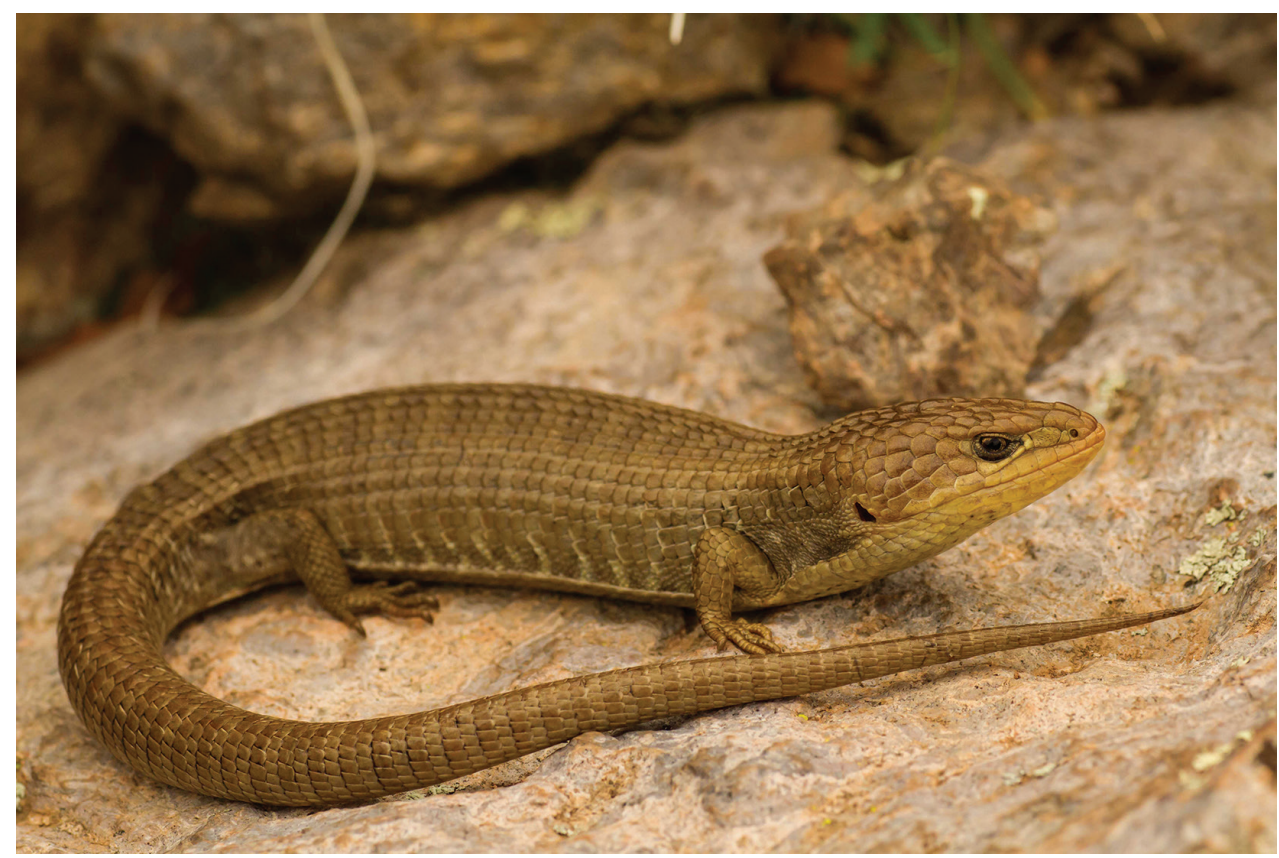

Figure 4. Barisia levicollis (female). Sierra del Nido, Chihuahua. Photo courtesy of Marisa Ishimatsu.

and Apalone spinifera). The remaining two species of turtles are widely distributed from southern Canada to northern Mexico (Chrysemys picta) and from southeastern Sonora, through the Pacific Coast, to Costa Rica (Rhinoclemmys pulcherrima).

Fifteen of the 51 species of lizards that occur in Chihuahua are endemic to Mexico, two of them to the state of Chihuahua (Barisia levicollis [Fig. 4] and Plestiodon multilineatus), one of the remaining 13 endemics is limited to the Bolsón de Mapimí (Uma paraphygas), one more to a small area in eastern Sonora and western Chihuahua (Sceloporus lemosespinali), another one to the temperate forests of western Chihuahua and northern Durango (Plestiodon bilineatus), one more to the Chihuahua Desert from northern Chihuahua to central Mexico (Holbrookia approximans), two others occupy areas in the Sierra Madre Occidental and the Sierra Madre Oriental (Barisia ciliaris), and even the Transvolcanic Belt (Phrynosoma orbiculare). The remaining seven endemic species are distributed mainly along the Pacific Coast of Mexico (Anolis nebulosus, Ctenosaura macrolopha, Sceloporus albiventris, S. nelsoni, Urosaurus bicarinatus, Plestiodon parviauriculatus, and Aspidoscelis costata).

The remaining 36 lizard species are not endemic to Mexico, one of them is distributed from southern Canada to northern Mexico (Phrynosoma hernandesi), and two more range from Mexico to northern Guatemala (Heloderma horridum) or to Costa Rica (Phyllodactylus tuberculosus) mainly on the Pacific Coast. Another 32 are distributed in the United States and Mexico, most of them are species characteristic of the Chihuahua Desert or woodlands of the Sierra Madre Occidental (Elgaria kingii, Gerrhonotus infernalis, Crotaphytus collaris, Gambelia wislizenii, Coleonyx brevis, Cophosaurus texanus, 


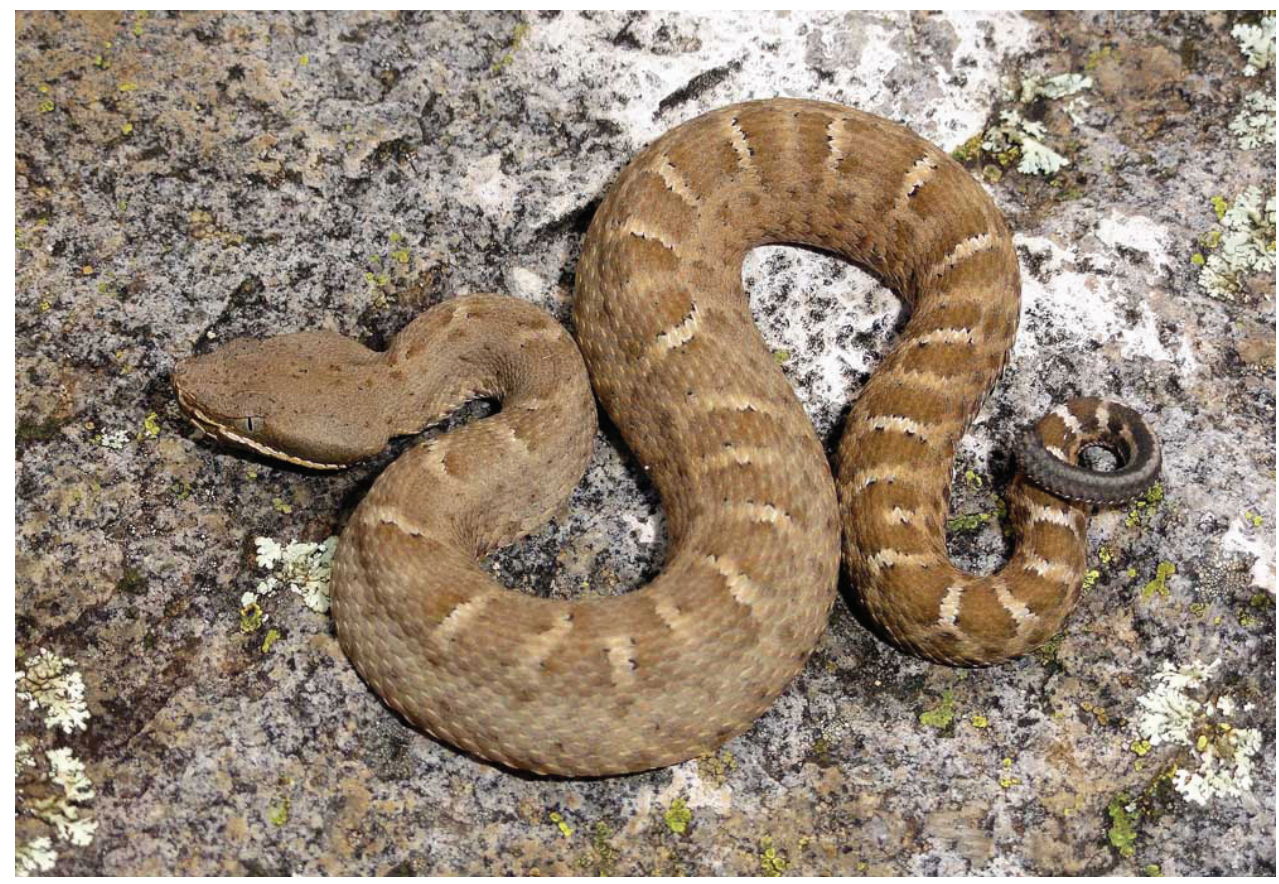

Figure 5. Crotalus willardi. Sierra del Nido, Chihuahua. Photo courtesy of Robert Bryson.

Holbrookia elegans, H. maculata, Phrynosoma cornutum, P. modestum, Sceloporus bimaculosus, S. clarkii, S. consobrinus, S. cowlesi, S. jarrovii, S. merriami, S. poinsettii, S. slevini, S. virgatus, Urosaurus ornatus, Uta stansburiana, Plestiodon callicephalus, P. multivirgatus, P. obsoletus, P. tetragrammus, Aspidoscelis exsanguis, A. gularis, A. inornata, A. marmorata, A. sonorae, $A$. tesselata, and $A$. uniparens). Only one of the 51 lizard species that occur in Chihuahua is an introduced species (Hemidactylus turcicus).

Twenty-one of the 73 species of snakes are endemic to Mexico (Conopsis nasus, Leptophis diplotropis, Mastigodryas cliftoni, Pituophis deppei, Salvadora bairdii, Sonora aemula, Sympholis lippiens, Trimorphodon tau, Geophis dugesii, Lampropeltis polyzona, Leptodeira splendida, Rhadinaea hesperia, R. laureata, Tropidodipsas repleta, Micrurus distans, Storeria storerioides, Thamnophis errans, T. melanogaster, T. unilabialis, T. validus, and Crotalus basiliscus). Thirty-seven snake species that are found in Chihuahua are distributed from the United States to Mexico (Arizona elegans, Bogertophis subocularis, Gyalopion canum, G. quadrangulare, Lampropeltis getula, L. knoblochi, Masticophis bilineatus, M. flagellum, M. taeniatus, Pantherophis emoryi, Rhinocheilus lecontei, Salvadora deserticola, S. grahamiae, Sonora semiannulata, Tantilla hobartsmithi, T. nigriceps, T. wilcoxi, T. yaquia, Trimorphodon vilkinsonii, Heterodon kennerlyi, Hypsiglena chlorophaea, H. jani, Micruroides euryxanthus, Rena dissecta, R. humilis, R. segrega, Nerodia erythrogaster, Thamnophis eques, Agkistrodon contortrix, Crotalus atrox, $C$. lepidus, $C$. molossus, C. ornatus, C. pricei, C. scutulatus, C. viridis, and C. willardi [Fig. 5]). Another four species range from northern Mexico to Central or even South America 
(Boa sigma, Masticophis mentovarius, Imantodes gemmistratus, and Agkistrodon bilineatus). Six more species are found from central or southern United States to Central or South America (Drymarchon melanurus, Drymobius margaritiferus, Oxybelis aeneus, Senticolis triaspis, Thamnophis cyrtopsis, and T. marcianus). Five more range from Canada to northern or central Mexico (Opheodrys vernalis, Pituophis catenifer, Diadophis punctatus, Thamnophis elegans, and T. sirtalis).

In terms of habitat types, 47 species are found in the temperate forests of the Sierra Madre Occidental. Forty-four are found in the subtropical canyons of the Sierra Madre Occidental. Fifty-eight species are found in the Chihuahuan Desert. One species is found in SON. Twenty-five species occupy more than one habitat type (i.e., are generalists).

\section{Likely species and poorly documented species}

There are several additional species that are likely to occur in Chihuahua, but that have not been recorded within the state. Three species of anurans might occur in the deep canyons and lowlands of the extreme southwestern part of the state. The Pacific Stream Frog (Craugastor vocalis) was recorded by Hardy and McDiarmid (1969) in extreme northeastern Sinaloa, $16 \mathrm{~km}$ NNE Choix, $520 \mathrm{~m}$, near the state line with Chihuahua. The Sabinal Frog (Leptodactylus melanonotus) was recorded by Bogert and Oliver (1945) from Güirocoba and Álamos, Sonora, only about 25 and $35 \mathrm{~km}$ respectively from the Chihuahua border, and by Smith et al. (2005) from the Río Mayo at the gates of Presa Mocuzari, Sonora. Hardy and McDiarmid (1969) mapped localities for this species (as L. occidentalis, a junior synonym) from throughout the lowlands of Sinaloa, including a locality in the extreme northeastern corner. The Lowland Burrowing Treefrog (Smilisca fodiens) has been recorded close to Chihuahua by Hardy and McDiarmid (1969) for Sinaloa, Bogert and Oliver (1945) for Sonora, and Trueb (1969) and Duellman (2001) for both states. Another anuran species likely to occur in extreme northeastern Chihuahua is the Gulf Coast Toad (Incilius nebulifer). This species of toad is represented by isolated populations at the southern extremity of the Big Bend region of Texas, adjacent to Coahuila (Conant and Collins 1998).

It is likely that at least four other turtle species occur in Chihuahua. Three species have been taken close to the state line with Sonora and Sinaloa, in the extreme southwestern part of the state. Kinosternon alamosae has been taken in the vicinity of Álamos, Sonora, about $35 \mathrm{~km}$ from the Chihuahua border. Trachemys hiltoni has been recorded from Güirocoba, $-25 \mathrm{~km}$ from Chihuahua, and from extreme northern Sinaloa (Hardy and McDiarmid 1969). Seidel (2002) mapped its range into Chihuahua, but only conjecturally. Legler and Webb (1970) stated that the species is limited to the Río El Fuerte drainage. These last authors stated that Trachemys yaquia is limited to the drainages of the Río Mayo, Río Sonora and Río Yaqui, however, Seidel (2002) conjectured that the range of this species extended into Chihuahua. In addition, the Common Snapping Turtle (Chelydra serpentina) occurs in the Río Grande at least in 
New Mexico (Degenhardt et al. 1996), and may well occur farther south in extreme northeastern Chihuahua, where little turtle trapping has been done.

There are at least nine lizard species not yet recorded in the state of Chihuahua that are likely to occur in it; four of them in the deep canyons and lowlands of extreme southwestern Chihuahua; three in the extreme northeastern part of the state; and two in the extreme northwestern part. The Zebra-tailed Lizard (Callisaurus draconoides) was recorded by Bogert and Oliver (1945) from Güirocoba and Álamos, Sonora ( $\sim 25$ and $35 \mathrm{~km}$ respectively from the Chihuahua border), and Hardy and McDiarmid (1969) spotted it at several localities in extreme northeastern Sinaloa. The Black Banded Gecko (Coleonyx fasciatus) has been recorded from five localities along the foothills of the Sierra Madre Occidental of eastern Sonora, three of these localities are in the Álamos region, one fairly close to Chihuahua. Its habitat suggests that it might occur in some of the deep canyons of southwestern Chihuahua. The Regal Horned Lizard (Phrynosoma solare) ranges from southern Arizona through almost all of Sonora, into northern Sinaloa. Hardy and McDiamid (1969) and Bogert and Oliver (1945) recorded it near Chihuahua in both Sinaloa and Sonora. It is a species of arid and semiarid habitats on plains, hills, and low mountain slopes. The Desert Spiny Lizard (Sceloporus magister) shows a range similar to that of the preceding species. East of the Sea of Cortés, it is the western representative of the eastern $S$. bimaculosus.

In northeastern Chihuahua the presence of three additional lizard species is likely. Wright (1971) indicated that the New Mexico Whiptail (Aspidoscelis neomexicana) is known from only central New Mexico and extreme southwestern Texas; almost all records are from near the Río Grande. He projected its range into Chihuahua along the Río Grande; although there are no records, its occurrence is highly likely there. Conant and Collins (1998) depicted the southern part of the Big Bend region of Texas as part of the range of the Reticulate Banded Gecko (Coleonyx reticulatus). It may be expected in adjacent parts of Chihuahua. Also Conant and Collins (1998) projected the range of the Texas Alligator Lizard (Gerrhonotus infernalis) to include the southern part of the Big Bend region of Texas, southward through eastern Chihuahua, most of Coahuila and other states to the south. In northwestern Chihuahua the presence of the Western Banded Gecko (Coleonyx variegatus) is expected. As indicated in Stebbins (2003) and Degenhardt et al. (1996), this species occurs in extreme southwestern New Mexico, and probably also in adjacent northwestern Chihuahua. The Gila Monster (Heloderma suspectum) is also expected to occur in this part of the state. The known occurrence of this species in Sonora, Arizona, and New Mexico close to the Chihuahua border indicates that occurrence in Chihuahua is likely.

It is highly likely that nine more snake species occur within the state of Chihuahua. Two of them in southwestern Chihuahua (Phyllorhynchus browni and Pseudoficimia frontalis); four in northeastern Chihuahua (Coluber constrictor, Lampropeltis alterna, Pantherophis bairdi, Tantilla cucullata); two in the northwestern part of the state (Crotalus tigris, Sistrurus catenatus); and one in extreme southeastern Chihuahua (Tantilla atriceps). The Saddled Leaf-nosed Snake (Phyllorhynchus browni) was recorded by Bogert and Oliver (1945) from Alamos, $-35 \mathrm{~km}$ from the Chihuahua border; Hardy 
(1972) reviewed the distribution of The False Ficimia (Pseudoficimia frontalis), citing specimens from near Álamos and Güirocoba, Sonora, -35 and $25 \mathrm{~km}$ from the Chihuahua border, respectively. The North American Racer (Coluber constrictor) is rare in Mexico, with only three records. Two are from Coahuila, including one from the extreme northwestern corner, in the Sierra del Carmen (Wilson 1966). Occurrence in Chihuahua seems likely. The Gray-banded Kingsnake (Lampropeltis alterna) is well known in the Big Bend of Texas, and elsewhere in that state, as well as in Coahuila and other adjacent states in Mexico, but it has never been found in Chihuahua, although it almost certainly occurs there. Baird's Ratsnake (Pantherophis bairdi) occurs in western Texas, including the Big Bend region, as well as northern Coahuila (Conant and Collins 1998); it is highly likely to occur in adjacent Chihuahua. The Trans-Pecos Blackheaded Snake (Tantilla cucullata) is known only in Texas, in the Big Bend and immediate vicinity (Dixon et al. 2000); occurrence in adjacent Coahuila and Chihuahua is to be expected. In northwestern Chihuahua the occurrence of the Tiger Rattlesnake (Crotalus tigris) is expected. Stebbins (2003) indicates occurrence of this species in the extreme southeastern corner of Arizona, and in eastern Sonora near the Chihuahua border. An inhabitant of arid and semiarid foothills deserts, it may enter the latter state in some of its semiarid valleys. Another rattlesnake, the Massasagua (Sistrurus catenatus), is known from southern New Mexico (Degenhardt et al. 1996) and southeastern Arizona (Brennan and Holycross 2006); it likely occurs in adjacent Chihuahua. In extreme southeastern Chihuahua the occurrence of the Mexican Black-headed Snake (Tantilla atriceps) is expected. The known range of this species comes close to the southeastern corner of the state (Cole and Hardy 1981, Conant and Collins 1998).

Some amphibian and reptile species are known to occur in Chihuahua from only a few records, including the Sonoran Desert Toad (Incilius alvarius) recorded by Santos-Barrera et al. (2006) in the municipality of Janos; the Spectacled Chirping Frog (Eleutherodactylus interorbitalis) recorded by Lemos-Espinal et al. (2006) in Cumbre del Caballo, Chínipas; the Cliff Chirping Frog (Eleutherodactylus marnockii) recorded by Lemos-Espinal et al. (2001) in the Grutas de Coyame; the Many-lined Skink (Plestiodon multivirgatus) recorded only by Van Devender and Van Devender (1975) at Ojo de Galeana; the Smooth Green Snake (Opheodrys vernalis) recorded only by Van Devender and Lowe (1977) at $38.4 \mathrm{~km}$ SE of Guerrero; the Crowned Graceful Brown Snake (Rhadinaea laureata) recorded by Villa et al. (2012) near km 86 on Hwy $25 \mathrm{~N}$ of Creel, Bocoyna, and $1 \mathrm{~km} \mathrm{~N}$ of Baborigame, Guadalupe y Calvo; the Banded Blacksnake (Tropidodipsas repleta) recorded by H. Smith and Lemos-Espinal (2006) at km 36 road Temoris-Chínipas, Guazapares; and the Plain-bellied Watersnake (Nerodia erythrogaster) recorded by Uriarte-Garzón and García-Vázquez (2014) in the municipality of Ojinaga.

\section{Comparisons with neighboring states}

Overall, the species of amphibians and reptiles in Chihuahua represent just over 37\% of the total pool of species from Chihuahua and its neighboring states (Tables 3, 4). 
Table 3. Total number of native amphibian and reptile species in each state arranged according to taxonomic order/suborder. Superscripts indicate number of introduced species to the state.

\begin{tabular}{l|c|c|c|c|c|c|c}
\hline \multicolumn{1}{c|}{ Order/Suborder } & Chihuahua & New Mexico & Texas & Sonora & Sinaloa & Durango & Coahuila \\
\hline Caudata & 4 & 3 & 28 & 3 & 1 & 3 & 4 \\
\hline Anura & $33^{1}$ & $23^{1}$ & $41^{1}$ & $33^{2}$ & 35 & $30^{1}$ & 20 \\
\hline Crocodilia & & & 1 & 1 & 1 & & \\
\hline Testudina & 13 & 10 & $30^{1}$ & $16^{1}$ & 12 & 5 & 11 \\
\hline Squamata/Lacertilia & $50^{1}$ & $46^{1}$ & $45^{6}$ & $66^{3}$ & 35 & $49^{1}$ & $49^{1}$ \\
\hline Squamata/Serpentes & 73 & 52 & $75^{2}$ & $71^{1}$ & 62 & $59^{1}$ & 49 \\
\hline TOTAL & $\mathbf{1 7 3}^{\mathbf{2}}$ & $\mathbf{1 3 4}^{\mathbf{2}}$ & $\mathbf{2 2 0}^{\mathbf{1 0}}$ & $\mathbf{1 9 0}^{\mathbf{7}}$ & $\mathbf{1 4 6}^{*}$ & $\mathbf{1 4 6}^{\mathbf{3}}$ & $\mathbf{1 3 3}^{\mathbf{1}}$ \\
\hline
\end{tabular}

*Introduced species for the state of Sinaloa are not documented in Enderson et al. (2009).

Species of reptiles from Chihuahua make up even more of the total pool of species, especially the Squamata, and more specifically Anguids and Snakes. Chihuahuan amphibians make up less of the species pool, especially salamanders. Chihuahua has a good proportion of the region's Ambystomatid salamanders, but is very depauperate in Plethodontids.

Overall, Chihuahua shares the highest proportion of its species with Sonora followed by Durango (Table 4). This is particularly evident in amphibians, with over $80 \%$ of Chihuahuan amphibians shared with Sonora. For reptiles, Chihuahua shares nearly $77 \%$ of its species with Durango and 66\% with Sonora. Chihuahua generally shares the least number of species with Coahuila, Sinaloa, and Texas. These patterns of shared species are likely a function of the extent to which these states share habitat types. For example, Chihuahua, Sonora, and Durango all have extensive desert habitats whereas Texas, for example, has a much more diverse range of habitats than Chihuahua. In addition, Sonora and Chihuahua share the habitats and species found in the Sierra Madre Occidental. Our results considering Chihuahua and all of its neighboring states parallels the results of an analysis of the states along the US-Mexico border using Jaccard hierarchical clustering analyses (Smith and Lemos-Espinal 2015).

\section{Conservation status}

Most of the herpetofauna of Chihuahua falls in the IUCNs least concern category (119 of 132 [does not include DD species]; 90\%), and as not listed by SEMARNAT (105 of 172; 61\%) (Table 2). These percentages are similar to those from other recently compiled tallies of conservation statuses for Mexican states (Coahuila: LemosEspinal and G. Smith 2016, Hidalgo: Lemos-Espinal and G. Smith 2015, Nayarit: Woolrich-Piña et al. 2016, Nuevo León: Lemos-Espinal et al. 2016). However, there are species of conservation concern in Chihuahua. For example, turtles and tortoises in Chihuahua appear to be a group of particular conservation concern with nearly half considered Vulnerable or Near Threatened by IUCN and more than half listed as $\operatorname{Pr}$ 
Table 4. Summary of the numbers of species shared between Chihuahua and neighboring Mexican and American states (not including introduced species). The percent of Chihuahuan species shared by a neighboring state are given in parentheses. Total refers to the total number of species found in Chihuahua and all the neighboring states (i.e., regional species pool) and the number in parentheses in this column is the percent of the regional species pool found in Chihuahua. -- indicates either Chihuahua or the neighboring state has no species in the taxonomic group, thus no value for shared species is provided.

\begin{tabular}{|c|c|c|c|c|c|c|c|c|}
\hline & Chihuahua & $\begin{array}{c}\text { New } \\
\text { Mexico }\end{array}$ & Texas & Sonora & Sinaloa & Durango & Coahuila & Total \\
\hline Class Amphibia & 37 & $17(45.9)$ & $17(45.9)$ & $30(81.1)$ & $20(54.0)$ & $23(62.2)$ & $15(40.5)$ & $122(30.1)$ \\
\hline Order Caudata & 4 & $1(25)$ & $1(25)$ & $3(75)$ & $1(25)$ & $3(75)$ & $1(25)$ & $36(11.1)$ \\
\hline Ambystomatidae & 3 & $1(33.3)$ & $1(33.3)$ & $2(66.7)$ & $1(33.3)$ & $3(100)$ & $1(33.3)$ & $8(37.5)$ \\
\hline Amphiumidae & 0 & - & - & - & - & - & - & $1(0)$ \\
\hline Plethodontidae & 1 & $0(0)$ & $0(0)$ & $1(100)$ & - & - & - & $22(4.5)$ \\
\hline Proteidae & 0 & - & - & - & - & - & - & $1(0)$ \\
\hline Salamandridae & 0 & - & - & - & - & - & - & $2(0)$ \\
\hline Sirenidae & 0 & - & - & - & - & - & - & $2(0)$ \\
\hline Order Anura & 33 & $16(48.5)$ & $16(48.5)$ & $27(81.8)$ & $19(57.6)$ & $20(60.6)$ & $14(42.4)$ & $86(38.4)$ \\
\hline Bufonidae & 10 & $6(60)$ & $6(60)$ & $9(90)$ & $6(60)$ & $8(80)$ & $6(60)$ & $21(47.6)$ \\
\hline Craugastoridae & 2 & $1(50)$ & $1(50)$ & $2(100)$ & $1(50)$ & $2(100)$ & (50) & $5(40)$ \\
\hline Eleutherodactylidae & 2 & - & $1(50)$ & $1(50)$ & $1(50)$ & $0(0)$ & $1(50)$ & $10(20)$ \\
\hline Hylidae & 5 & $2(40)$ & $2(40)$ & $5(100)$ & $4(80)$ & $3(60)$ & $2(40)$ & $22(22.7)$ \\
\hline Leptodactylidae & 0 & - & - & - & - & - & - & $2(0)$ \\
\hline Microhylidae & 3 & $1(33.3)$ & $2(66.7)$ & $2(66.7)$ & $2(66.7)$ & $1(33.3)$ & $1(33.3)$ & $5(60)$ \\
\hline Ranidae & 8 & $3(37.5)$ & $1(12.5)$ & $6(75)$ & $4(50)$ & $4(50)$ & $1(12.5)$ & $16(50)$ \\
\hline Rhinophryni & 0 & - & - & - & - & - & - & 1( \\
\hline Scaphiopodidae & 3 & $3(100)$ & $3(100)$ & $2(66.7)$ & $1(33.3)$ & $2(66.7)$ & $2(66.7)$ & $4(75)$ \\
\hline Class Reptilia & 136 & $76(55.9)$ & 66 (48.5) & $90(66.2)$ & $61(44.8)$ & $86(76.8)$ & $62(59.6)$ & 343 (39.6) \\
\hline Order Crocodylia & 0 & - & - & - & - & - & - & $2(0)$ \\
\hline Crocodylidae & 0 & - & - & - & - & - & - & $2(0)$ \\
\hline Order Testudines & 13 & $6(46.2)$ & $6(46.2)$ & $6(46.2)$ & $4(30.8)$ & $5(38.5)$ & $6(46.2)$ & $47(27.6)$ \\
\hline Chelonidae & 0 & - & - & - & - & - & - & $5(0)$ \\
\hline Chelydridae & 0 & - & - & - & - & - & - & $2(0)$ \\
\hline Dermochelyidae & 0 & - & - & - & - & - & - & $1(0)$ \\
\hline Emydidae & 4 & $3(75)$ & $3(75)$ & $2(50)$ & $1(25)$ & $1(25)$ & $1(25)$ & $22(18.2)$ \\
\hline Geomydidae & 1 & - & - & $1(100)$ & $1(100)$ & - & - & $1(100)$ \\
\hline Kinosternidae & 5 & $2(40)$ & $2(40)$ & & & $3(60)$ & $3(60)$ & $10(50)$ \\
\hline Testudinidae & 2 & - & $0(0)$ & $1(50)$ & $1(50)$ & $1(50)$ & $1(50)$ & $4(5)$ \\
\hline Trionychidae & 1 & $1(100)$ & $1(100)$ & - & - & - & $1(100)$ & $2(50)$ \\
\hline Order Squamata & 123 & $70(56.9)$ & $60(48.8)$ & $84(68.3)$ & 57 (46.3) & $81(65.8)$ & $56(45.5)$ & 294 (41.8) \\
\hline Suborder Lacertilia & 50 & $30(60)$ & $25(50)$ & $33(66)$ & $17(34)$ & $34(68)$ & $23(46)$ & $143(35)$ \\
\hline Anguidae & 4 & $1(25)$ & $1(25)$ & $1(25)$ & $1(25)$ & $3(75)$ & $2(50)$ & $7(57.1)$ \\
\hline Crotaphytidae & 2 & $2(100)$ & $2(100)$ & $2(100)$ & - & $2(100)$ & $2(100)$ & $6(33.3)$ \\
\hline Dactyloidae & 1 & - & $0(0)$ & $1(100)$ & $1(100)$ & $1(100)$ & - & $4(25)$ \\
\hline Eublepharidae & 1 & $1(100)$ & $1(100)$ & $0(0)$ & $0(0)$ & $1(100)$ & $1(100)$ & $4(25)$ \\
\hline Helodermatidae & 1 & $0(0)$ & - & $1(100)$ & $1(100)$ & $1(100)$ & - & $2(50)$ \\
\hline Iguanidae & 1 & - & - & $1(100)$ & $1(100)$ & $0(0)$ & - & $9(11.1)$ \\
\hline Phrynosomatidae & 24 & $16(66.7)$ & $12(50)$ & $19(79.2)$ & $9(37.5)$ & $18(75)$ & $12(50)$ & $61(39.3)$ \\
\hline
\end{tabular}




\begin{tabular}{l|c|c|c|c|c|c|c|c}
\hline & Chihuahua & $\begin{array}{c}\text { New } \\
\text { Mexico }\end{array}$ & Texas & Sonora & Sinaloa & Durango & Coahuila & Total \\
\hline Phyllodactylidae & 1 & - & - & $1(100)$ & $1(100)$ & $1(100)$ & - & $5(20)$ \\
\hline Scincidae & 7 & $3(42.8)$ & $3(42.8)$ & $3(42.8)$ & $2(28.6)$ & $3(42.8)$ & $2(28.6)$ & $18(39.9)$ \\
\hline Teiidae & 8 & $7(87.5)$ & $6(75)$ & $4(50)$ & $1(12.5)$ & $4(50)$ & $4(50)$ & $23(34.8)$ \\
\hline Xantusidae & 0 & - & - & - & - & - & - & $4(0)$ \\
\hline Suborder Serpentes & 73 & $40(54.8)$ & $35(47.9)$ & $51(69.9)$ & $40(54.8)$ & $47(64.4)$ & $33(45.2)$ & $151(48.3)$ \\
\hline Boidae & 1 & - & - & $1(100)$ & $1(100)$ & $1(100)$ & - & $2(50)$ \\
\hline Colubridae & 35 & $21(60)$ & $19(54.3)$ & $24(68.6)$ & $22(62.8)$ & $25(71.4)$ & $17(48.6)$ & $66(53)$ \\
\hline Dipsidae & 10 & $4(40)$ & $3(30)$ & $7(70)$ & $6(60)$ & $6(60)$ & $3(30)$ & $22(45.4)$ \\
\hline Elapidae & 2 & $1(50)$ & $0(0)$ & $2(100)$ & $2(100)$ & - & $0(0)$ & $4(50)$ \\
\hline Leptotyphlopidae & 3 & $2(66.7)$ & $2(66.7)$ & $1(33.3)$ & $1(33.3)$ & $1(33.3)$ & $2(66.7)$ & $4(75)$ \\
\hline Natricidae & 11 & $6(54.5)$ & $4(36.4)$ & $7(63.6)$ & $3(27.3)$ & $9(81.8)$ & $3(27.3)$ & $32(34.4)$ \\
\hline Viperidae & 11 & $6(54.5)$ & $7(63.6)$ & $9(81.8)$ & $5(45.4)$ & $5(745.4)$ & $8(72.7)$ & $21(52.4)$ \\
\hline TOTAL & $\mathbf{1 7 3}$ & $\mathbf{9 3}$ & $\mathbf{8 3}$ & $\mathbf{1 2 0}$ & $\mathbf{8 1}$ & $\mathbf{1 0 9}$ & $\mathbf{7 7}(\mathbf{4 4 . 5 )}$ & $\mathbf{4 6 5 ( 3 7 . 2 )}$ \\
\hline
\end{tabular}

or A by SEMARNAT. Emydidae and Testudinae are the families of most conservation concern. Indeed, turtles account for 4 of the 13 species (31\%) of the Chihuahuan herpetofauna that are categorized as Vulnerable, Near Threatened, or Endangered by the IUCN, even though they make up only $7 \%$ of the species in Chihuahua. We also found that turtles as a group also have the highest mean Environmental Vulnerability Score (EVS), especially Emydidae, Testudinidae, and Trionychidae. We therefore encourage additional emphasis be placed on better understanding the ecology and conservation status of turtle and tortoise populations in Chihuahua.

In addition, even though there are relatively few reptiles and amphibians placed on conservation lists in Chihuahua, this does not mean they are safe. Indeed, there are species, such as Craugastor tarahumaraensis, Ctenosaura macrolopha, Uma paraphygas, and Tropidodipsas repleta that are of great conservation concern based on their EVS values (Wilson et al. 2013a, b). In addition, the more locally appropriate EVS assessments (see Wilson et al. 2013a,b) also suggest that conservation concern should exist for the amphibian families Ambystomatidae, Craugastoridae, and Eleutherodactylidae; and the non-turtle reptile families Anguidae, Eublepharidae, Iguanidae, Scincidae, Teiidae, and Elapidae.

Even beyond these species and families, the environment and habitats of Chihuahua are subject to anthropogenic change, such as construction of border fences (Lasky et al. 2011), increased urbanization (Biggs et al. 2010), and changes in precipitation and increased drying associated with climate change (Seager and Vecchi 2010). Indeed, the distribution of species at high risk according to the EVS assessment $(\geq 14$; Wilson et al. 2013a,b) is not the same across habitat types. Nearly $40 \%$ of species $(18 / 47)$ in the temperate forests of the Sierra Madre Occidental is at risk according to the EVS, and nearly a third of species in the Chihuahuan Desert (19/58). Just over 20\% (10/44) of species in subtropical canyons of the Sierra Madre Occidental are at high risk. Generalist species (those that use more than one habitat type) are the least at risk ( 2 of 25 species). These results suggest that particular conservation attention should be paid to 
the Sierra Madre Occidental and the Chihuahuan Desert habitat types in Chihuahua. We thus again emphasize that continued and increased study of the herpetofauna of Chihuahua is needed to monitor the possible effects of any environmental changes.

\section{Acknowledgments}

We thank Louis Porras, Jesús Sigala, and one anonymous reviewer for their helpful comments on a previous version of this manuscript. Andrew Gottscho helped us with the map for the State of Chihuahua.

\section{References}

Acevedo AA, Lampo M, Cipriani R (2016) The Cane or Marine Toad, Rhinella marina (Anura: Bufonidae): two genetically and morphologically distinct species. Zootaxa 4103: 574-586. https://doi.org/10.11646/zootaxa.4103.6.7

Alvarado-Díaz J, Suazo-Ortuño I, Wilson LD, Medina-Aguilar O (2013) Patterns of physiographic distribution and conservation status of the herpetofauna of Michoacán, Mexico. Amphibian \& Reptile Conservation 7: 128-170.

Anderson CG, Greenbaum E (2012) Phylogeography or northern populations of the Blacktailed Rattlesnakes (Crotalus molossus Baird and Girard, 1853), with the revalidation of $C$. ornatus Hallowell, 1854. Herpetological Monographs 26: 19-57. https://doi.org/10.1655/ HERPMONOGRAPHS-D-11-00012.1

Biggs TW, Atkinson E, Powell R, Ojeda-Revah L (2010) Land cover following rapid urbanization on the US-Mexico border: Implications for conceptual models of urban watershed processes. Landscape and Urban Planning 96: 78-87. https://doi.org/10.1016/j.landurbplan.2010.02.005

Bogert CM, Oliver JA (1945) A preliminary analysis of the herpetofauna of Sonora. Bulletin of the American Museum of Natural History 83: 297-426.

Brennan TC, Holycross AT (2006) A Field Guide to Amphibians and Reptiles in Arizona. Arizona Game and Fish Department. Phoenix, AZ, 150 pp.

Carbajal-Márquez RA, Quintero Díaz GE (2016) The herperofauna of Aguascalientes, México. Revista Mexicana de Herpetología 2: 1-30.

Card DC, Schied DR, Adams RH, Corbin AB, Perry BW, Andrew AL, Pasquesi GI, Smith EN, Jezkova T, Boback SM, Booth W, Castoe TA (2016) Phylogeographic and population genetic analyses reveal multiple species of Boa and independent origins of insular dwarfism. Molecular Phylogenetics and Evolution 102: 104-116. https://doi.org/10.1016/j. ympev.2016.05.034

Cole CJ, Hardy LM (1981) Systematics of North American colubrid snakes related to Tantilla planiceps (Blainville). Bulletin of the American Museum of Natural History 171: 199-284.

Conant R, Collins JT (1998) A Field Guide to Reptiles and Amphibians: Eastern and Central North America. Third edition, expanded. Houghton Mifflin, Boston, 616 pp. 
Degenhardt WG, Painter CW, Price AH (1996) Amphibians and reptiles of New Mexico. University of New Mexico Press, Albuquerque, $431 \mathrm{pp}$.

Dixon JR (2015) Herpetofauna of Texas. In: Lemos-Espinal JA (Ed.) Amphibians and Reptiles of the US-Mexico Border States. Texas A\&M University Press, College Station, 181-195.

Dixon JR, Lemos-Espinal JA (2010) Anfibios y Reptiles del Estado de Querétaro, México/Amphibians and Reptiles of the State of Querétaro, Mexico. CONABIO, 428 pp.

Dixon JR, Vaughan RK, Wilson LD (2000) The taxonomy of Tantilla rubra and allied taxa (Serpentes: Colubridae). Southwestern Naturalist 45: 141-153. https://doi.org/10.2307/3672455

Duellman WE (2001) Hylid frogs of Middle America. Revised and expanded edition. Society for the Study of Amphibians and Reptiles, Ithaca.

Edwards T, Karl A, Vaughn M, Rosen P, Meléndez-Torres C, Murphy R (2016) The desert tortoise trichotomy: Mexico hosts a third, new sister-species of tortoise in the Gopherus morafkai-G. agassizii group. ZooKeys 562: 131-158. https://doi.org/10.3897/zookeys. 562.6124

Enderson EF, Turner DS, Rosen PC, Bezy RL (2009) The herpetofauna of Sonora, Mexico, with comparisons to adjoining states. Check List 5: 632-672. https://doi.org/10.15560/5.3.632

Farr W (2015) Herpetofauna of Tamaulipas. In: Lemos-Espinal JA (Ed.) Amphibians and Reptiles of the US-Mexico Border States. Texas A\&M University Press, College Station, 101-121.

Frost DR (2016) Amphibian Species of the World: an Online Reference. Version 6.0. American Museum of Natural History, New York. http://research.amnh.org/herpetology/amphibia/ index.html [June 30, 2016]

Grismer LL (2002) Amphibians and Reptiles of Baja California, including its Pacific Islands and the Islands of the Sea of Cortés. University of California Press, Berkeley, 413 pp. https://doi.org/10.1525/california/9780520224179.001.0001

Hardy LM (1972) A systematic revision of the genus Pseudoficimia (Serpentes: Colubridae). Journal of Herpetology 6: 53-69. https://doi.org/10.2307/1563094

Hardy LM, McDiarmid RW (1969) The amphibians and reptiles of Sinaloa, Mexico. University of Kansas Publications, Museum of Natural History 18: 39-252. https://doi.org/10.5962/ bhl.part. 19990

IUCN (2016) IUCN Red List of Threatened Species, Version 2016.1. IUCN 2016, IUCN Red List of Threatened Species.

INEGI [Instituto Nacional de Estadística y Geografía] (2001) Modelo Digital de Terreno. Escala 1: 250,000. INEGI, México.

INEGI (Instituto Nacional de Geografía, Estadística e Informática - Dirección General de Geografía) (2004) Anuario Estadístico Chihuahua. INEGI, México, 632 pp.

Johnson JD, Mata-Silva V, García-Padilla E, Wilson LD (2015b) The herpetofauna of Chiapas, Mexico: composition, distribution, and conservation. Mesoamerican Herpetology 2: 272-329.

Johnson JD, Mata-Silva V, Wilson LD (2015a) A conservation reassessment of the Central American herpetofauna based on the EVS measure. Amphibian \& Reptile Conservation 9: 1-94.

Lasky JR, Jetz W, Keitt TH (2011) Conservation biogeography of the US-Mexico border: a transcontinental risk assessment of barriers to animal dispersal. Diversity and Distributions 17: 673-687. https://doi.org/10.1111/j.1472-4642.2011.00765.x 
Lee JC (1996) The amphibians and reptiles of the Yucatan Peninsula. Cornell University Press, Ithaca, $500 \mathrm{pp}$.

Legler JM, Webb RG (1970) A new slider turtle (Pseudemys scripta) from Sonora, Mexico. Herpetologica 26: 27-37.

Lemos-Espinal JA (2015) Amphibians and Reptiles of the US-Mexico Border States. Texas A\&M University Press, College Station, 614 pp.

Lemos-Espinal JA, Dixon JR (2013) Amphibians and Reptiles of San Luis Potosí. Eagle Mountain Publishing, LC. Eagle Mountain, Utah, 312 pp.

Lemos-Espinal JA, Rorabaugh JC (2015) Herpetofauna of Sonora. In: Lemos-Espinal JA (Ed.)

Amphibians and Reptiles of the US-Mexico Border States. Texas A\&M University Press, College Station, 34-51.

Lemos-Espinal JA, Smith GR (2015) Amphibians and reptiles of the state of Hidalgo, Mexico. Check List 11(3): 1642. https://doi.org/10.15560/11.3.1642

Lemos-Espinal JA, Smith GR (2016) Amphibians and Reptiles of the State of Coahuila, Mexico, with comparison with adjoining states. ZooKeys 593: 117-137. https://doi.org/10.3897/ zookeys.593.8484

Lemos-Espinal JA, Smith GR, Cruz A (2016) Amphibians and reptiles of the state of Nuevo León, Mexico. ZooKeys 594: 123-141. https://doi.org/10.3897/zookeys.594.8289

Lemos-Espinal JA, Smith HM (2007a) Anfibios y Reptiles del Estado de Chihuahua, México/ Amphibians and Reptiles of the State of Chihuahua, Mexico. CONABIO, México, 628 pp. Lemos-Espinal JA, Smith HM (2007b) Anfibios y Reptiles del Estado de Coahuila, México/ Amphibians and Reptiles of the State of Coahuila, Mexico. CONABIO, México, 563 pp. Lemos-Espinal JA, Smith HM (2015a) Herpetofauna of Chihuahua. In: Lemos-Espinal JA (Ed.) Amphibians and Reptiles of the US-Mexico Border States. Texas A\&M University Press, College Station, 52-65.

Lemos-Espinal JA, Smith HM (2015b) Herpetofauna of Coahuila. In: Lemos-Espinal JA (Ed.) Amphibians and Reptiles of the US-Mexico Border States. Texas A\&M University Press, College Station, 66-82.

Lemos-Espinal JA, Smith HM, Chiszar D (2001) Geographic distribution: Eleutherodactylus marnockii. Herpetological Review 32: 270.

Lemos-Espinal JA, Smith HM, Chiszar D (2006) Syrrhophus interorbitalis (Amphibia: Anura) in Chihuahua, Mexico. Bulletin of the Maryland Herpetological Society 32: 176-178.

Lemos-Espinal JA, Smith HM, Cruz A (2013) Amphibians and Reptiles of the Sierra Tarahumara of Chihuahua, Mexico. ECO Herpetological Publishing and Distribution. Rodeo, New Mexico, USA, 405 pp.

Lemos-Espinal JA, Smith HM, Dixon JR, Cruz A (2015) Anfibios y reptiles de Sonora, Chihuahua y Coahuila, México / Amphibians and reptiles of Sonora, Chihuahua, and Coahuila, Mexico. CONABIO. Tomo / Volume I, 714 pp; Tomo / Volume II, 668 pp.

Martin PS, Yetman D, Fishbein M, Jenkins P, Van Devender TR, Wilson RK (1998) Gentry's Río Mayo Plants. University of Arizona Press, Tucson, 557 p.

Mata-Silva V, Johnson JD, Wilson LD, García-Padilla E (2015) The herpetofauna of Oaxaca, Mexico: composition, physiographic distribution, and conservation status. Mesoamerican Herpetology 2: 5-62. 
McCranie JR, Wilson LD (2001) The herpetofauna of the Mexican State of Aguascalientes. Courier Forschungsinstitut Senckenberg 230: 1-57.

Nevárez-De Los Reyes M, Lazcano D, Banda-Leal J (2016) Geographic Distribution: Crotalus ornatus (Eastern Black-tailed Rattlesnake). Herpetological Review 47: 261

Owen R (1844) Characters of a new species of Axolotl. Annual Magazine of Natural History 15, No. 88, 23 pp.

Painter C, Stuart J (2015) Herpetofauna of New Mexico. In: Lemos-Espinal JA (Ed.) Amphibians and Reptiles of the US-Mexico Border States. Texas A\&M University Press, College Station, 164-180.

Ramírez-Bautista A, Hernández-Salinas U, García-Vázquez UO, Leyte-Manrique A, CansecoMárquez L (2009) Herpetofauna del Valle de México: Diversidad y Conservación. CONABIO, México.

Rorabaugh JC (2008) An introduction to the herpetofauna of mainland Sonora, Mexico, with comments on conservation and management. Journal of the Arizona-Nevada Academy of Science 40(1):20-65. https://doi.org/10.2181/1533-6085(2008)40[20:AITTHO]2.0.CO;2

Rorabaugh J, Lemos-Espinal JA (2016) A Field Guide to the Amphibians and Reptiles of Sonora, Mexico. ECO Herpetological Publishing and Distribution, Rodeo, New Mexico, 688 pp.

Santos-Barrera G, Cruzado J, Ponce-Guevara E, Sierra-Corona R, Pacheco J (2006) Geographic distribution: Bufo alvarius. Herpetological Review 37: 58.

Santos-Barrera G, Flores-Villela O (2011) A new species of toad of the genus Incilius from the Sierra Madre Occidental of Chihuahua, Mexico (Anura: Bufonidae). Journal of Herpetology 45: 211-215. https://doi.org/10.1670/10-093.1

Seager R, Vecchi GA (2010) Greenhouse warming and the $21^{\text {st }}$ century hydroclimate of southwestern North America. Proceedings of the National Academy of Sciences 107: 21277-21282. https://doi.org/10.1073/pnas.0910856107

Seidel ME (2002) Taxonomic observations on extant species and subspecies of slider turtles, genus Trachemys. Journal of Herpetology 36: 285-292. https://doi.org/10.1670/00221511(2002)036[0285:TOOESA]2.0.CO;2

SEMARNAT (Secretaría de Medio Ambiente y Recursos Naturales) (2010) Norma Oficial Mexicana NOM-059-Ecol-2010. Protección ambiental-Especies nativas de México de flora y fauna silvestres-Categorías de riesgo y especificaciones para su inclusión, exclusión o cambio-Lista de especies en riesgo. Diario oficial (Segunda Sección, 30-dic), 77 pp.

Smith GR, Lemos-Espinal JA (2015) Herpetofaunal diversity of the United States-Mexico Border States, with comparison among the states. In: Lemos-Espinal JA (Ed.) Amphibians and Reptiles of the US-Mexico Border States. Texas A\&M University Press, College Station, 196-205.

Smith HM, Lemos-Espinal JA (2006) A second specimen and first record for Chihuahua, Mexico, of Tropidodipsas repleta (Serpentes: Colubridae). Bulletin of the Maryland Herpetological Society 42: 171-172.

Smith HM, Lemos-Espinal JA, Chiszar D (2005) Amphibians and Lizards from Sonora, Chihuahua and Coahuila. Bulletin of the Chicago Herpetological Society 40: 45-51.

Stebbins RC (2003) A field guide to western reptiles and amphibians. Third edition. Houghton Mifflin, Boston, 533 pp. 
Tanner WW (1985) Snakes of western Chihuahua. Great Basin Naturalist 45: 615-676. https:// doi.org/10.5962/bhl.part.4437

Terán-Juárez SA, García-Padilla E, Mata-Silva V, Johnson JD, Wilson LD (2016) The herpetofauna of Tamaulipas, Mexico: composition, distribution, and conservation status. Mesoamerican Herpetology 3: 42-113

Trueb L (1969) Pternohyla Boulenger. Burrowing tree frogs. Catalogue of American Amphibians and Reptiles 77: 1-4.

Uetz P, Hošek J (2015) The Reptile Database. http://www.reptile-database.org [December 6, 2015] Uriarte-Garzón P, García-Vázquez UO (2014) Primer registro de Nerodia erytrogaster bogerti (Conant, 1963) (Serpentes: Colubridae) para el estado de Chihuahua, México. Acta Zoologica Mexicana 30(1): 221-225.

Valdez-Lares R, Muñiz Martínez R, Gadsden H, Aguirre León G, Castañeda Gaytán G, González Trápaga R (2013) Checklist of amphibians and reptiles of the state of Durango, México. Check List 9: 714-724. https://doi.org/10.15560/9.4.714

Van Devender TR, Lowe C (1977) Amphibians and reptiles of Yepomera, Chihuahua, Mexico. Journal of Herpetology 11:41-50. https://doi.org/10.2307/1563290

Van Devender T, Van Devender W (1975) Ecological notes on two Mexican skinks. Southwestern Naturalist 20: 279-282. https://doi.org/10.2307/3670449

Villa AR, Bryson RW, Ramírez-Chaparro R (2012) Geographic Distribution Notes: Rhadinaea laureata: Chihuahua. Herpetological Review 43: 107.

Wilson LD (1966) The range of the Rio Grande racer in Mexico and the status of Coluber oaxaca (Jan). Herpetologica 22: 42-47.

Wilson LD, Johnson JD, Mata-Silva V (2013a) A conservation reassessment of the amphibians of Mexico based on the EVS measure. Amphibian \& Reptile Conservation 7(1): 97-127.

Wilson LD, Mata-Silva V, Johnson JD (2013b) A conservation reassessment of the reptiles of Mexico based on the EVS measure. Amphibian \& Reptile Conservation 7(1): 1-47.137

Woolrich-Piña GA, Ponce-Campos P, Loc-Barragán J, Ramírez-Silva JP, Mata-Silva V, Johnson JD, García-Padilla E, Wilson LD (2016) The herpetofauna of Nayarit, Mexico: composition, distribution, and conservation. Mesoamerican Herpetology 3: 376-448.

Wyndham FS (2004) Learning Ecology: Ethnobotany in the Sierra Tarahumara, Mexico. Ph.D. thesis, Athens, USA: University of Georgia.

Wright JW (1971) Cnemidophorus neomexicanus. Catalogue of American Amphibians and Reptiles 109: $1-3$. 